\title{
Factorization of the hypergeometric-type difference equation on the non-uniform lattices: dynamical algebra
}

\author{
R. Álvarez-Nodarse ${ }^{\dagger \ddagger}$, N. M. Atakishiyev ${ }^{\S}$ and R. S. Costas-Santos* \\ † Departamento de Análisis Matemático. \\ Universidad de Sevilla. Apdo. 1160, E-41080 Sevilla, Spain \\ ¥ Instituto Carlos I de Física Teórica y Computacional, \\ Universidad de Granada, E-18071 Granada, Spain \\ § Instituto de Matemáticas, UNAM, Apartado Postal 273-3, \\ C.P. 62210 Cuernavaca, Morelos, México \\ * Departamento de Matemáticas, E.P.S., Universidad Carlos III de Madrid. \\ Ave. Universidad 30, E-28911, Leganés, Madrid, Spain
}

14th October 2004

\begin{abstract}
We argue that one can factorize the difference equation of hypergeometric type on the nonuniform lattices in general case. It is shown that in the most cases of $q$-linear spectrum of the eigenvalues this directly leads to the dynamical symmetry algebra $s u_{q}(1,1)$, whose generators are explicitly constructed in terms of the difference operators, obtained in the process of factorization. Thus all models with the $q$-linear spectrum (some of them, but not all, previously considered in a number of publications) can be treated in a unified form.
\end{abstract}

\section{Introduction and preliminaries}

In this paper we continue the study, started in [1], on the factorization of the hypergeometrictype difference equation on the non-uniform lattices, i.e., of the equation [2]

$$
\begin{gathered}
\sigma(s) \frac{\Delta}{\Delta x\left(s-\frac{1}{2}\right)}\left[\frac{\nabla y(s)}{\nabla x(s)}\right]+\tau(s) \frac{\Delta y(s)}{\Delta x(s)}+\lambda y(s)=0, \\
\sigma(s)=\tilde{\sigma}(x(s))-\frac{1}{2} \tilde{\tau}(x(s)) \Delta x\left(s-\frac{1}{2}\right), \quad \tau(s)=\tilde{\tau}(x(s)),
\end{gathered}
$$

where $\Delta y(s):=y(s+1)-y(s), \nabla y(s):=y(s)-y(s-1), \tilde{\sigma}(x(s))$ and $\tilde{\tau}(x(s))$ are polynomials in $x(s)$ of degree at most 2 and 1 , respectively, and $\lambda$ is a constant (see also [3]). The difference equation (1) has polynomial solutions $P_{n}(x(s) ; q):=P_{n}(s ; q)$ of the hypergeometric type if and only if the lattice $x(s)$ has the form $[4,5]$

$$
x(s)=c_{1}(q) q^{s}+c_{2}(q) q^{-s}+c_{3}(q)=c_{1}(q)\left[q^{s}+q^{-s-\mu}\right]+c_{3}(q),
$$

where $c_{1}, c_{2}, c_{3}$ and $q^{\mu}:=c_{1} / c_{2}$ are constants which, in general, depend on $q$. An important special case of the lattice $x(s)$ is the $q$-linear lattice, which is obtained from (2) by assuming that either $c_{1}(q)$ or $c_{2}(q)$ vanishes. 
The polynomial solutions of the difference equation (1) correspond to the following expression [3] for its eigenvalues $\lambda_{n}(q)$ :

$$
\begin{gathered}
\lambda_{n}(q)=C_{1} q^{n}+C_{2} q^{-n}+C_{3}, \\
C_{1}=\frac{1}{2(1-q)}\left(\widetilde{\tau}^{\prime}+\frac{\widetilde{\sigma}^{\prime \prime}}{k_{q}}\right), \quad C_{2}=\frac{1}{2\left(1-q^{-1}\right)}\left(\widetilde{\tau}^{\prime}-\frac{\widetilde{\sigma}^{\prime \prime}}{k_{q}}\right), \quad C_{3}=-\frac{\widetilde{\sigma}^{\prime \prime}(1+q)}{2 k_{q}(1-q)}-\frac{\widetilde{\tau}^{\prime}}{2},
\end{gathered}
$$

where $\widetilde{\tau}^{\prime}$ and $\widetilde{\sigma}^{\prime \prime}$ are the coefficients of $x(s)$ and $x^{2}(s)$ in the Taylor expansion for $\tilde{\tau}(x(s))$ and $\tilde{\sigma}(x(s))$, respectively, i.e., $\tilde{\tau}(x(s))=\widetilde{\tau}^{\prime} x(s)+\widetilde{\tau}(0)$, and $\tilde{\sigma}(x(s))=\widetilde{\sigma}^{\prime \prime} / 2 x^{2}(s)+\widetilde{\sigma}^{\prime}(0) x(s)+\tilde{\sigma}(0)$.

Observe that the coefficients $C_{1}$ and $C_{2}$ of the $q^{n}$ and $q^{-n}$ terms, respectively, are fixed by the functions $\sigma$ and $\tau$ in (1), and so is the product $C_{1} C_{2}$. In what follows we denote by $L_{q}$ the value of $C_{1} C_{2}=\left(\left(\widetilde{\sigma}^{\prime \prime} / k_{q}\right)^{2}-\left(\widetilde{\tau}^{\prime}\right)^{2}\right) / 4 k_{q}^{2}$.

The sequence $\left\{\lambda_{n}(q)\right\}$ satisfies the following three-term recurrence relation (TTRR)

$$
\lambda_{n+2}(q)-\left(q+q^{-1}\right) \lambda_{n+1}(q)+\lambda_{n}(q)=\frac{1}{2}\left(\tilde{\tau}^{\prime} k_{q}^{2}-\tilde{\sigma}^{\prime \prime}[2]_{q}\right)=C .
$$

Conversely, if $\left\{\lambda_{n}(q)\right\}$ satisfy the TTRR (4), then it has the form $\lambda_{n}(q)=C_{1}^{\prime} q^{n}+C_{2}^{\prime} q^{-n}+C_{3}^{\prime}$. Obviously, having used the initial conditions $\lambda_{0}(q)=0$ and $\lambda_{1}(q)=-\widetilde{\tau}^{\prime}$, one recovers the expression (3).

It is well known [3] that under certain conditions the polynomial solutions of (1) are orthogonal. For example, if $\left.\sigma(s) \rho(s) x^{k}\left(s-\frac{1}{2}\right)\right|_{s=a, b}=0$, for all $k=0,1,2, \ldots$, then the polynomial solutions possess a discrete orthogonality property

$$
\sum_{s=a}^{b-1} P_{n}(s ; q) P_{m}(s ; q) \rho(s) \nabla x_{1}(s)=d_{n}^{2}(q) \delta_{n, m},
$$

where the weight function $\rho(s)$ is a solution of the Pearson-type difference equation [3]

$$
\frac{\Delta}{\Delta x\left(s-\frac{1}{2}\right)}[\sigma(s) \rho(s)]=\tau(s) \rho(s) \quad \text { or } \quad \sigma(s+1) \rho(s+1)=\sigma(-s-\mu) \rho(s) .
$$

If the lattice $x(s)$ is a $q$-linear lattice, i.e., $x(s)=c q^{ \pm s}+c_{3}$, then the $\sigma(-s-\mu)$ in $(6)$ should be substituted by $\sigma(s)+\tau(s) \Delta x(s-1 / 2)$. A more detailed information on orthogonal polynomials on the non-uniform lattices can be found in $[3,5,6,7,8]$.

In [1] it has been shown that one can factorize the Nikiforov-Uvarov equation (1) with the aid of raising and lowering operators, which can be constructed for solutions of this equation. In this paper we wish to make one step further by studying the dynamical symmetry algebra for the hypergeometric-type difference equation (1) on the non-uniform lattices (2). Our approach is essentially based on the simple observation, formulated in [9]: In order to factorize an arbitrary difference equation, one should express it explicitly in terms of the shift (or displacement) operators $\exp \left(a \frac{d}{d s}\right)$, which are defined as $\exp \left(a \frac{d}{d s}\right) f(s)=f(s+a), a$ is some constant. For example, in the case of the equation (1) this corresponds to the substitutions $\Delta=\exp \left(\frac{d}{d s}\right)-1$ and $\nabla=1-\exp \left(-\frac{d}{d s}\right)$. This procedure converts a difference equation into an eigenvalue problem for a difference operator, represented by a linear combination of some shift operators (with coefficients, which depend polynomially on the variable $s$ ). Since each term of this linear combination is readily factorizable (because $\exp (\alpha+\beta) A=\exp \alpha A \exp \beta A$ for an arbitrary operator $A$ ), the factorization of the whole linear combination, which represents the 
initial difference equation, becomes straightforward.

Inspired by the appearance of Macfarlane's [10] and Biedenharn's [11] important constructions of $q$-analogues of quantum harmonic oscillator, this technique of factorization of difference equations was later employed in a number of publications [12]-[16] in order to study group theoretic properties of the various well-known families of orthogonal polynomials, which can be viewed as $q$-extensions of the classical Hermite polynomials. So our purpose here is to formulate a unified approach to deriving all of these results, which correspond to the $q$-linear spectrum.

An important aspect to observe at this point is that we shall mainly (except for the examples in subsection 4.2) confine our attention to those families of $q$-polynomials, which satisfy discrete orthogonality relation of the type (5). The explanation of such preference is that the factorization of difference equations for instances of $q$-polynomials with continuous orthogonality property has been already thoroughly studied in [12]-[16]. Observe also that our approach still remains valid in the limit as $q \rightarrow 1$; so classical counterparts of $q$-polynomials, which will be discussed in this paper, are in fact incorporated as appropriate limit cases. But the reader who desires to know more about the factorization in the cases of classical orthogonal polynomials (such as the Kravchuk, Charlier, Meixner, Meixner-Pollaczek, and Hahn) may be referred to $[17,18]$ and references therein.

The paper is organized as follows. In section 2 we associate with each family of $q$-polynomials a " $q$-Hamiltonian" $\mathfrak{H}(s ; q)$ (via the second-order difference equation) and construct two difference operators $a(s ; q)$ and $b(s ; q)$, which factorize the operator $\mathfrak{H}(s ; q)$. Our main results are given in section 3: they are formulated in Theorems 3.4 - which gives a simple necessary and sufficient condition that the $q$-Hamiltonian $\mathfrak{H}(s ; q)$ admits the factorization in terms of the operators $a(s ; q)$ and $b(s ; q)$, which satisfy the relation $a(s ; q) b(s ; q)-q^{\gamma} b(s ; q) a(s ; q)=I$ for some $\gamma$, and 3.5 - stating that the eigenvalues of the difference equation (1) in this case should be of the form $\lambda_{n}(q)=C_{1} q^{n}+C_{3}$ or $\lambda_{n}(q)=C_{2} q^{-n}+C_{3}$. In section 4 several relevant examples of particular $q$-families of orthogonal polynomials are illustrated.

\section{Factorization operators}

Let introduce a set of functions $\Phi_{n}$

$$
\Phi_{n}(s ; q)=d_{n}^{-1} A(s) \sqrt{\rho(s)} P_{n}(s ; q),
$$

where $d_{n}$ is the norm of the $q$-polynomials $P_{n}(s ; q), \rho(s)$ is the solution of the Pearson equation (6) and $A(s)$ is an arbitrary continuous function, $A(s) \neq 0$ in the interval $(a, b)$ of orthogonality of $P_{n}$. If the polynomials $P_{n}(s ; q)$ possess the discrete orthogonality property (5), then the functions $\Phi_{n}(s ; q)$ satisfy

$$
\left\langle\Phi_{n}(s ; q), \Phi_{m}(s ; q)\right\rangle=\sum_{s=a}^{b-1} \Phi_{n}(s ; q) \Phi_{m}(s ; q) \frac{\nabla x_{1}(s)}{A^{2}(s)}=\delta_{n, m} .
$$

Notice that if $A(s)=\sqrt{\nabla x_{1}(s)}$, then the set $\left(\Phi_{n}\right)_{n}$ is an orthonormal set. Obviously, in the case of a continuous orthogonality (as for the Askey-Wilson polynomials) one needs to change the sum in (8) by a Riemann integral $[3,5]$.

Next, we define the $q$-Hamiltonian $\mathfrak{H}(s ; q)$ of the form

$$
\mathfrak{H}(s ; q):=\frac{1}{\nabla x_{1}(s)} A(s) H(s ; q) \frac{1}{A(s)},
$$


where

$$
H(s ; q):=-\frac{\sqrt{\sigma(-s-\mu+1) \sigma(s)}}{\nabla x(s)} e^{-\partial_{s}}-\frac{\sqrt{\sigma(-s-\mu) \sigma(s+1)}}{\Delta x(s)} e^{\partial_{s}}+\left(\frac{\sigma(-s-\mu)}{\Delta x(s)}+\frac{\sigma(s)}{\nabla x(s)}\right) I,
$$

$e^{\alpha \partial s} f(s)=f(s+\alpha)$ for all $\alpha \in \mathbb{C}$ and $I$ is the identity operator. If we now use the identity $\nabla=\Delta-\nabla \Delta$ and the equation (1), we find that

$$
\mathfrak{H}(s ; q) \Phi_{n}(s ; q)=\lambda_{n} \Phi_{n}(s ; q),
$$

i.e., the functions $\Phi_{n}(s ; q)$, defined in $(7)$, are the eigenfunctions of the associated operator $\mathfrak{H}(s ; q)$.

Our first step is to find two operators $a(s ; q)$ and $b(s ; q)$ such that the Hamiltonian $\mathfrak{H}(s ; q)=$ $b(s ; q) a(s ; q)$, i.e., the operators $a(s ; q)$ and $b(s ; q)$ factorize the $q$-Hamiltonian $\mathfrak{H}(s ; q)$. But before exhibiting their explicit form let us point out that if there exists a pair of such operators, then there are infinitely many of them. Indeed, let $a(s ; q)$ and $b(s ; q)$ be such operators that $\mathfrak{H}(s ; q)=b(s ; q) a(s ; q)$ and let $U(s ; q)$ be an arbitrary unitary operator, i.e., $U^{\dagger}(s ; q) U(s ; q)=I$. Then the operators

$$
\widetilde{a}(s ; q):=U(s ; q) a(s ; q), \quad \widetilde{b}(s ; q):=b(s ; q) U^{\dagger}(s ; q),
$$

also factorize $\mathfrak{H}(s ; q)$ for

$$
\widetilde{b}(s ; q) \widetilde{a}(s ; q)=b(s ; q) U^{\dagger}(s ; q) U(s ; q) a(s ; q)=b(s ; q) I a(s ; q)=b(s ; q) a(s ; q)=\mathfrak{H}(s ; q) .
$$

This arbitrariness in picking up a particular unitary operator $U(s)$ is very essential because it enables one to construct a closed algebra, which contains a Hamiltonian $\mathfrak{H}(s ; q)$ itself. An explicit form of the spectrum of this Hamiltonian may then be found by purely algebraic arguments from the knowledge of representations of this algebra (which is therefore referred to as $a$ dynamical algebra).

If one applies the standard factorization procedure to the equation (1), then the following difference operators emerge

Definition 2.1 Let $\alpha$ be a real number and $A(s)$ an arbitrary continuous non-vanishing function in $(a, b)$. We define a family of $\alpha$-down and $\alpha$-up operators by

$$
\begin{aligned}
& \mathfrak{a}_{\alpha}^{\downarrow}(s ; q):=\frac{A(s)}{\sqrt{\nabla x_{1}(s)}} e^{-\alpha \partial_{s}}\left(e^{\partial_{s}} \sqrt{\frac{\sigma(s)}{\nabla x(s)}}-\sqrt{\frac{\sigma(-s-\mu)}{\Delta x(s)}}\right) \frac{1}{A(s)}, \\
& \mathfrak{a}_{\alpha}^{\uparrow}(s ; q):=\frac{1}{\nabla x_{1}(s)} A(s)\left(\sqrt{\frac{\sigma(s)}{\nabla x(s)}} e^{-\partial_{s}}-\sqrt{\frac{\sigma(-s-\mu)}{\Delta x(s)}}\right) e^{\alpha \partial_{s}} \frac{\sqrt{\nabla x_{1}(s)}}{A(s)}
\end{aligned}
$$

respectively.

A straightforward calculation (by using the simple identity $e^{\partial_{s}} \nabla=\Delta$ ) shows that for all $\alpha \in \mathbb{R}$

$$
\mathfrak{H}(s ; q)=\mathfrak{a}_{\alpha}^{\uparrow}(s ; q) \mathfrak{a}_{\alpha}^{\downarrow}(s ; q),
$$

i.e., the operators $\mathfrak{a}_{\alpha}^{\downarrow}(s ; q)$ and $\mathfrak{a}_{\alpha}^{\uparrow}(s ; q)$ factorize the Hamiltonian, defined in (9). i.e., we have the following

Theorem 2.2 Given a q-Hamiltonian (9) $\mathfrak{H}(s ; q)$, then the operators $\mathfrak{a}_{\alpha}^{\downarrow}(s ; q)$ and $\mathfrak{a}_{\alpha}^{\uparrow}(s ; q)$ defined in (12) are such that for all $\alpha \in \mathbb{C}, \mathfrak{H}(s ; q)=\mathfrak{a}_{\alpha}^{\uparrow}(s ; q) \mathfrak{a}_{\alpha}^{\downarrow}(s ; q)$. 
Our next step is to find a dynamical algebra, associated with the Hamiltonian $\mathfrak{H}(s ; q)$. To this end we will need the following definition

Definition 2.3 A function $f(z)$ is said to be a linear-type function of $z$, if there exist two functions $F$ and $G$, such that for all $z, \zeta \in \mathbb{C}$, this function $f(z)$ can be represented as

$$
f(z+\zeta)=F(\zeta) f(z)+G(\zeta) .
$$

A particular case of the linear-type functions are the $q$-linear functions, i.e., the functions of the form $f(z)=A q^{z}+B$. For these functions $f(z+\zeta)=F(\zeta) f(z)+G(\zeta)$, where $F(\zeta)=q^{\zeta}$ and $G(\zeta)=B\left(1-q^{\zeta}\right)$.

Remark 2.4 If we use the expression in (3) for the eigenvalues $\lambda_{n}$, then it is straightforward to see that $\lambda_{n}$ is a q-linear function of $n$ if and only if $\widetilde{\sigma}^{\prime \prime}= \pm k_{q} \widetilde{\tau}^{\prime}$. Moreover, in this case we have

$$
\widetilde{\sigma}^{\prime \prime}=k_{q} \widetilde{\tau}^{\prime} \Rightarrow \lambda_{n}(q,+)=\frac{\widetilde{\tau}^{\prime}}{1-q}\left(q^{n}-1\right), \quad \text { and } \quad \tilde{\sigma}^{\prime \prime}=-k_{q} \widetilde{\tau}^{\prime} \Rightarrow \lambda_{n}(q,-)=\frac{\widetilde{\tau}^{\prime}}{1-q^{-1}}\left(q^{-n}-1\right) .
$$

Notice that $\lambda_{n}(q,-)=\lambda_{n}\left(q^{-1},+\right)$, i.e., the second case can be obtained form the first one just by changing $q$ to $q^{-1}$.

Proposition 2.5 The function $\lambda_{n}$ is a q-linear function of $n$ if and only if it satisfies $\lambda_{n+1}=$ $q \lambda_{n}+C$.

Proof: A straightforward computations show that if $\lambda_{n}$ is a $q$-linear function of $n$, then it satisfies the recurrence formula $\lambda_{n+1}=q \lambda_{n}+C$, where $C$ is a constant (in this case $C=\lambda_{1}$ ). But the general solution of the difference equation $\lambda_{n+1}=q \lambda_{n}+C$ is $\lambda_{n}=A q^{n}+D$, where $A$ and $D$ are, in general, non-vanishing constants.

Remark 2.6 Notice that if $\lambda_{n}$ is a q-linear function of $n$, then $\lambda_{n}$ satisfies the recurrence relation $\lambda_{n+\gamma}-q^{\gamma} \lambda_{n}=C$ for any numbers $\gamma$ and $C$.

Finally, we have the following straightforward lemma

Lemma 2.7 Let $x(s)$ be a q-linear function of $s$ and $\lambda_{n}$ be the eigenvalue of the difference equation of hypergeometric type (1). Then $\lambda_{n}$ is a q-linear function of $n$ if and only if $\Delta^{(2)}(\sigma(s))=0$ and $q^{-1}$-linear function of $n$ if and only if $\Delta^{(2)}(\sigma(-s-\mu))=0$, where $\Delta^{(2)}$ is the operator $\Delta^{(2)}=\frac{\Delta}{\Delta x_{1}(s)} \frac{\Delta}{\Delta x(s)}$.

Proof: It follows from equation (14) and the fact that $\Delta^{(2)}(\sigma(s))=\frac{[2]_{q}}{2}\left(\widetilde{\sigma}^{\prime \prime}-\tilde{\tau}^{\prime} k_{q}\right)$ and $\Delta^{(2)}(\sigma(-s-\mu))=\frac{[2]_{q}}{2}\left(\widetilde{\sigma}^{\prime \prime}+\tilde{\tau}^{\prime} k_{q}\right)$.

\section{Dynamical algebra}

We begin this section with the following definition.

Definition 3.1 Let $\varsigma$ be a real number, and let $a(s ; q)$ and $b(s ; q)$ be two operators. We define the $\varsigma$-commutator of $a$ and $b$ as

$$
[a(s ; q), b(s ; q)]_{\varsigma}=a(s ; q) b(s ; q)-\varsigma b(s ; q) a(s ; q) .
$$


Proposition 3.2 Let $\mathfrak{H}(s ; q)$ be an operator, such that there exist two operators $a(s ; q)$ and $b(s ; q)$ and two real numbers $\varsigma$ and $\Lambda$, such that $\mathfrak{H}(s ; q)=b(s) a(s ; q)$, and $[a(s ; q), b(s ; q)]_{\varsigma}=\Lambda$. Then, if $\Phi(s ; q)$ is an eigenvector of the Hamiltonian $\mathfrak{H}(s ; q)$, associated with the eigenvalue $\lambda$, we have

1. $\mathfrak{H}(s ; q)\{a(s ; q) \Phi(s ; q)\}=\varsigma^{-1}(\lambda-\Lambda)\{a(s ; q) \Phi(s ; q)\}$, i.e., a $(s ; q) \Phi(s ; q)$ is the eigenvector of $\mathfrak{H}(s ; q)$, associated with the eigenvalue $\varsigma^{-1}(\lambda-\Lambda)$,

2. $\mathfrak{H}(s ; q)\{b(s ; q) \Phi(s ; q)\}=(\Lambda+\varsigma \lambda)\{b(s ; q) \Phi(s ; q)\}$, i.e., $b(s ; q) \Phi(s ; q)$ is the eigenvector of $\mathfrak{H}(s ; q)$, associated with the eigenvalue $\Lambda+\varsigma \lambda$.

Proof: In the first case, since $\mathfrak{H}(s ; q) \Phi(s ; q)=\lambda \Phi(s ; q)$,

$$
\begin{aligned}
\mathfrak{H}(s ; q)\{a(s ; q) \Phi(s ; q)\} & =b(s ; q) a(s ; q)\{a(s ; q) \Phi(s ; q)\}=\varsigma^{-1}(a(s ; q) b(s ; q)-\Lambda)\{a(s ; q) \Phi(s ; q)\} \\
& =\varsigma^{-1}(\lambda-\Lambda)\{a(s ; q) \Phi(s ; q)\} .
\end{aligned}
$$

By the same token, in the second case

$$
\begin{aligned}
\mathfrak{H}(s ; q)\{b(s ; q) \Phi(s ; q)\} & =b(s ; q) a(s ; q) b(s ; q) \Phi(s ; q)=b(s ; q)(\Lambda+\varsigma \lambda) \Phi(s ; q) \\
& =(\Lambda+\varsigma \lambda)\{b(s ; q) \Phi(s ; q)\} .
\end{aligned}
$$

In the same way one can prove that

$$
a(s ; q) b(s ; q) \Phi(s ; q)=(\Lambda+\varsigma \lambda) \Phi(s ; q) .
$$

Moreover, if $\Phi(s ; q)$ is an eigenvector of the Hamiltonian $\mathfrak{H}(s ; q)$ (or of the operator $a(s ; q) b(s ; q)$ ), then $a^{k}(s ; q) \Phi(s ; q)$ and $b^{k}(s ; q) \Phi(s ; q)$ are, in general, also eigenvectors.

Remark 3.3 Obviously, the condition $[a(s ; q), b(s ; q)]_{\varsigma}=I$ can be changed to $[a(s ; q), b(s ; q)]_{\varsigma}=$ $\Lambda$, where $\Lambda$ is an arbitrary non-zero constant. In fact, if the operators $a(s ; q)$ and $b(s ; q)$ satisfy the $q$-commutation relation $[a(s ; q), b(s ; q)]_{\varsigma}=\Lambda$, then the operators $\mathfrak{a}(s ; q)=\Lambda^{-1 / 2} a(s ; q)$ and $\mathfrak{b}(s ; q)=\Lambda^{-1 / 2} b(s ; q)$ satisfy $[\mathfrak{a}(s ; q), \mathfrak{b}(s ; q)]_{\varsigma}=I$, and $\mathfrak{H}(s ; q)=\Lambda \mathfrak{b}(s ; q) \mathfrak{a}(s ; q)$.

The Proposition 3.2 thus refers to the case of a system, described by a Hamiltonian $\mathfrak{H}(s ; q)$, which admits the factorization (13) in terms of the operators $a(s ; q)$ and $b(s ; q)$, satisfying the $q$-commutation relation $[a(s ; q), b(s ; q)]_{\varsigma}=I$. Moreover, it tells us how to construct a dynamical symmetry algebra for such a case in a direct fashion [19]. Indeed, let us assume that $[a(s ; q), b(s ; q)]_{\varsigma}=I, \varsigma=q^{2}\left(\right.$ or $\left.q^{-2}\right)$, and $b(s ; q)=a^{\dagger}(s ; q)$. Then one can rewrite the $q^{2}$ commutator $a(s ; q) a^{\dagger}(s ; q)-q^{2} a^{\dagger}(s ; q) a(s ; q)=I$ in the following form

$$
\left[a(s ; q), a^{\dagger}(s ; q)\right]:=a(s ; q) a^{\dagger}(s ; q)-a^{\dagger}(s ; q) a(s ; q)=I-\left(1-q^{2}\right) a^{\dagger}(s ; q) a(s ; q):=q^{2 N(s)},
$$

where, by definition, the operator $N(s)$ is equal to $N(s)=\ln \left[I-\left(1-q^{2}\right) a^{\dagger}(s ; q) a(s ; q)\right] / \ln q^{2}$. From this definition of $N(s)$ it follows that

$$
[N(s), a(s ; q)]=-a(s ; q), \quad\left[N(s), a^{\dagger}(s ; q)\right]=a^{\dagger}(s ; q),
$$

i.e., $N(s)$ is the number operator. The next (and final) step is to introduce a new set of the operators

$$
b(s ; q):=q^{-N(s) / 2} a(s ; q), \quad b^{\dagger}(s ; q):=a^{\dagger}(s ; q) q^{-N(s) / 2},
$$

which satisfy the following commutation relation

$$
b(s ; q) b^{\dagger}(s ; q)-q b^{\dagger}(s ; q) b(s ; q)=q^{-N(s)},
$$


readily derived with the aid of (16). The operators $b(s ; q), b^{\dagger}(s ; q)$, and $N(s)$ directly lead to the dynamical algebra $s u_{q}(1,1)$ with the generators

$$
K_{0}(s)=\frac{1}{2}[N(s)+1 / 2], \quad K_{+}(s)=\beta\left(b^{\dagger}(s ; q)\right)^{2}, \quad K_{-}(s)=\beta b^{2}(s ; q), \quad \beta^{-1}=q+q^{-1} .
$$

It is straightforward to verify that thus defined generators satisfy the standard commutation relations

$$
\left[K_{0}(s), K_{ \pm}(s)\right]= \pm K_{ \pm}(s), \quad\left[K_{-}(s), K_{+}(s)\right]=\left[2 K_{0}(s)\right]_{q^{2}}
$$

of the algebra $s u_{q}(1,1)$ (see e.g. [20]).

Thus in the case when the operators $a(s ; q)$ and $b(s ; q)$, which factorize the Hamiltonian $\mathfrak{H}(s ; q)$, satisfy the $q$-commutation relation $[a(s ; q), b(s ; q)]_{\varsigma^{2}}=I$ and $b(s ; q)=a^{\dagger}(s ; q)$, the appropriate dynamical symmetry algebra is $s u_{\varsigma}(1,1)$. So the question arises: what are conditions for insuring that such $q$-commutator takes place? In other words, we have the following

Problem 1: To find two operators $a(s ; q)$ and $b(s ; q)$ and a constant $\varsigma$ such that the Hamiltonian $\mathfrak{H}(s ; q)=b(s ; q) a(s ; q)$ and $[a(s ; q), b(s ; q)]_{\varsigma}=I$.

For the first part we already have the answer (see Theorem 2.2). The solution of the second one is formulated in the following two theorems.

Theorem 3.4 Let $\mathfrak{H}(s ; q)$ be the following difference operator (q-Hamiltonian)

$$
\mathfrak{H}(s ; q)=\frac{1}{\nabla x_{1}(s)} A(s) H(s ; q) \frac{1}{A(s)} .
$$

The operators $b(s ; q)=\mathfrak{a}_{\alpha}^{\uparrow}(s ; q)$ and $a(s ; q)=\mathfrak{a}_{\alpha}^{\downarrow}(s ; q)$ given in (12) factorize the Hamiltonian $\mathfrak{H}(s ; q)(17)$ and satisfy the commutation relation $[a(s ; q), b(s ; q)]_{\varsigma}=\Lambda$ for a certain real number $\varsigma$ if and only if the following two conditions hold:

$$
\frac{\nabla x(s)}{\nabla x_{1}(s-\alpha)} \sqrt{\frac{\nabla x_{1}(s-1) \nabla x_{1}(s)}{\nabla x(s-\alpha) \Delta x(s-\alpha)}} \sqrt{\frac{\sigma(s-\alpha) \sigma(-s-\mu+\alpha)}{\sigma(s) \sigma(-s-\mu+1)}}=\varsigma,
$$

and

$$
\frac{1}{\Delta x(s-\alpha)}\left(\frac{\sigma(s-\alpha+1)}{\nabla x_{1}(s-\alpha+1)}+\frac{\sigma(-s-\mu+\alpha)}{\nabla x_{1}(s-\alpha)}\right)-\varsigma \frac{1}{\nabla x_{1}(s)}\left(\frac{\sigma(s)}{\nabla x(s)}+\frac{\sigma(-s-\mu)}{\Delta x(s)}\right)=\Lambda .
$$

Proof: Taking the expression of the operators $a_{\alpha}^{\uparrow}(s)$ and $a_{\alpha}^{\downarrow}(s)$, a straightforward calculus shows that $a_{\alpha}^{\downarrow}(s) a_{\alpha}^{\uparrow}(s)=A_{1}(s) e^{\partial_{s}}+A_{2}(s) e^{-\partial_{s}}+A_{3}(s) I$, where

$$
\begin{aligned}
& A_{1}(s)=-\sqrt{\frac{\nabla x_{1}(s+1)}{\nabla x_{1}(s)}} \frac{A(s)}{A(s+1)} \sqrt{\frac{\sigma(s+1-\alpha) \sigma(-s-\mu-1+\alpha)}{\Delta x(s-\alpha) \Delta x(s+1-\alpha)}} \frac{1}{\nabla x_{1}(s+1-\alpha)}, \\
& A_{2}(s)=-\sqrt{\frac{\nabla x_{1}(s-1)}{\nabla x_{1}(s)}} \frac{A(s)}{A(s-1)} \sqrt{\frac{\sigma(s-\alpha) \sigma(-s-\mu+\alpha)}{\Delta x(s-1-\alpha) \Delta x(s-\alpha)}} \frac{1}{\nabla x_{1}(s-\alpha)}, \\
& A_{3}(s)=\frac{1}{\Delta x(s-\alpha)}\left[\frac{\sigma(s+1-\alpha)}{\nabla x_{1}(s+1-\alpha)}+\frac{\sigma(-s-\mu+\alpha)}{\nabla x_{1}(s-\alpha)}\right] .
\end{aligned}
$$


In the same way, using (10) and (9) we have $a_{\alpha}^{\uparrow}(s) a_{\alpha}^{\downarrow}(s)=\mathfrak{H}(s ; q)=B_{1}(s) e^{\partial_{s}}+B_{2}(s) e^{-\partial_{s}}+$ $B_{3}(s) I$, where

$$
\begin{aligned}
& B_{1}(s)=-\frac{1}{\nabla x_{1}(s)} \frac{A(s)}{A(s+1)} \frac{\sqrt{\sigma(-s-\mu) \sigma(s+1)}}{\nabla x(s+1)}, \\
& B_{2}(s)=-\frac{1}{\nabla x_{1}(s)} \frac{A(s)}{A(s-1)} \frac{\sqrt{\sigma(-s-\mu+1) \sigma(s)}}{\nabla x(s)}, \\
& B_{3}(s)=\frac{1}{\nabla x_{1}(s)}\left[\frac{\sigma(s)}{\nabla x(s)}+\frac{\sigma(-s-\mu)}{\Delta x(s)}\right] .
\end{aligned}
$$

Consequently,

$$
\left[a_{\alpha}^{\downarrow}(s), a_{\alpha}^{\uparrow}(s)\right]_{\varsigma}=\left(A_{1}(s)-\varsigma B_{1}(s)\right) e^{\partial_{s}}+\left(A_{2}(s)-\varsigma B_{2}(s)\right) e^{-\partial_{s}}+\left(A_{3}(s)-\varsigma B_{3}(s)\right) I .
$$

To eliminate the two terms in the right-hand side of (22), which are proportional to the difference operators $\exp \left( \pm \partial_{s}\right)$, one must require that

$$
A_{1}(s)-\varsigma B_{1}(s)=0, \quad A_{2}(s)-\varsigma B_{2}(s)=0 .
$$

Off hand, it is not evident that one can satisfy both of the relations (23), which only involve the same constant $\varsigma$. But it is straightforward to verify from (20) and (21) that

$$
A_{1}(s) B_{2}(s+1)=A_{2}(s+1) B_{1}(s),
$$

or, equivalently,

$$
\frac{A_{1}(s)}{B_{1}(s)}=\frac{A_{2}(s+1)}{B_{2}(s+1)}
$$

Hence, the requirement that $A_{1}(s)=\varsigma B_{1}(s)$ entails the relation $A_{2}(s)=\varsigma B_{2}(s)$, and vice versa. From (22) it is now evident that the commutator $\left[a_{\alpha}^{\uparrow}(s), a_{\alpha}^{\downarrow}(s)\right]_{\varsigma}$ is a constant if $(23)$ holds and the factor $A_{3}(s)-\varsigma B_{3}(s)$ is a constant. Thus, the required conditions (18) and (19) immediately follow.

Theorem 3.5 Let $\left(\Phi_{n}\right)_{n}$ the eigenfunctions of $\mathfrak{H}(s ; q)$ corresponding to the eigenvalues $\left(\lambda_{n}\right)_{n}$ and suppose that the problem 1 has a solution for $\Lambda \neq 0$. Then, the eigenvalues $\lambda_{n}$ of the difference equation (11) are q-linear or $q^{-1}$-linear functions of $n$, i.e., $\lambda_{n}=C_{1} q^{n}+C_{3}$ or $\lambda_{n}=C_{2} q^{-n}+C_{3}$, respectively.

Proof: ${ }^{1}$ In the following we use the notation $\varsigma=q^{\gamma}$. Suppose that problem 1 has a solution with $\Lambda \neq 0$ and $\lambda_{n}$ is not a $q$-linear (respectively, $q^{-1}$ ) function of $n$. From Proposition 3.2 we know that $\mathfrak{a}_{\alpha}^{\uparrow}(s ; q) \Phi_{n}(s ; q)$ is and eigenvector of $\mathfrak{H}(s ; q)$ corresponding to the eigenvalue $\Lambda+q^{\gamma} \lambda_{n}$. If we denote by $\Phi_{m(n) ; q}$ such eigenvector where $m(n)$ is a function of $n$, then we have $\Lambda+q^{\gamma} \lambda_{n}=\lambda_{m(n)}$. Then using (3) we get

$$
\lambda_{m(n)}=C_{1} q^{m(n)}+C_{2} q^{-m(n)}+C_{3}, \quad C_{1} C_{2}=L_{q} .
$$

On the other hand

$$
\lambda_{m(n)}=\Lambda+q^{\gamma} \lambda_{n}=C_{1} q^{\gamma} q^{n}+C_{2} q^{\gamma} q^{-n}+q^{\gamma} C_{3}+\Lambda=C_{1}^{\prime} q^{n}+C_{2}^{\prime} q^{-n}+C_{3}^{\prime} .
$$

But here, since $\lambda_{m(n)}$ is an eigenvalue of (1), again we have the condition $C_{1}^{\prime} C_{2}^{\prime}=L_{q}$, thus $C_{1} C_{2}=L_{q}=C_{1}^{\prime} C_{2}^{\prime}=C_{1} C_{2} q^{2 \gamma}$ so $q^{2 \gamma}=1$, i.e., $\gamma=0$, or $C_{1} C_{2}=0$. In the first case, equating

\footnotetext{
${ }^{1}$ For an alternative proof in the case $\alpha=0$ see the appendix.
} 
(24) and (25), we have that $C_{3}^{\prime}=C_{3} q^{\gamma}+\Lambda=C_{3}$, i.e., $\Lambda=0$ that is a contradiction. Thus $C_{1} C_{2}=0$ from where the result easily follows.

It is worth noting that the $q$-linearity of the eigenvalues of $\mathfrak{H}(s ; q)$ is only the necessary condition, i.e., it is not sufficient. So there are cases when the eigenvalues $\lambda_{n}(q)$ are $q$-linear (for instance, those which correspond to the $q$-Meixner, the $q$-Charlier, and the $q$-Laguerre polynomials with $a \neq q^{-1 / 2}$, but the corresponding $q$-Hamiltonians $\mathfrak{H}(s ; q)$ do not admit the factorization in terms of $q$-commuting operators. This just reflects the fact that an appropriate dynamical algebra is not $s u_{q}(1,1)$ and one has to consider a more complicated quadratic algebra $A W(3)$ [21]. The problem of finding an explicit connection between the generators of the algebra $A W(3)$ and the operators $a(s ; q)$ and $b(s ; q)$, which factorize the $q$-Hamiltonians for these cases, will be attended in a separate publication.

Remark 3.6 A special important case of the non-linear lattice is when $x(s)=\frac{1}{2}\left(q^{s}+q^{-s}\right)$. In this case if we put $\alpha=\frac{1}{2}$, then the conditions (18) and (19) of Theorem 3.4 becomes

$$
\sqrt{\frac{\sigma\left(s-\frac{1}{2}\right) \sigma\left(-s+\frac{1}{2}\right)}{\sigma(s) \sigma(-s+1)}}=\varsigma,
$$

and

$$
\frac{1}{\nabla x_{1}(s)}\left(\frac{\sigma\left(s+\frac{1}{2}\right)}{\Delta x(s)}+\frac{\sigma\left(-s+\frac{1}{2}\right)}{\nabla x(s)}\right)-\varsigma \frac{1}{\nabla x_{1}(s)}\left(\frac{\sigma(s)}{\nabla x(s)}+\frac{\sigma(-s)}{\Delta x(s)}\right)=\Lambda .
$$

respectively. Moreover, if we put $A(s)=1$ then, $\mathfrak{H}(s ; q)=\left(\nabla x_{1}(s)\right)^{-1} H(s ; q)$ and the $\alpha$-operators simplify

$$
\begin{aligned}
& a_{1 / 2}^{\downarrow}(s)=\frac{1}{\nabla x_{1}(s)}\left(e^{\frac{1}{2} \partial_{s}} \sqrt{\sigma(s)}-e^{-\frac{1}{2} \partial_{s}} \sqrt{\sigma(-s)}\right), \\
& a_{1 / 2}^{\uparrow}(s)=\frac{1}{\nabla x_{1}(s)}\left(\sqrt{\sigma(s)} e^{-\frac{1}{2} \partial_{s}}-\sqrt{\sigma(-s)} e^{\frac{1}{2} \partial_{s}}\right) .
\end{aligned}
$$

Now we can formulate the

Problem 2: To find two operators $a(s ; q)$ and $b(s ; q)$ and a constant $\varsigma$ such that the Hamiltonian $\mathfrak{H}(s ; q)=b(s ; q) a(s ; q)$ and $[a(s ; q), b(s ; q)]_{\varsigma}=I$ and such that $a(s ; q)$ and $b(s ; q)$ are the lowering and raising operators, i.e.,

$$
a(s ; q) \Phi_{n}(s ; q)=D_{n} \Phi_{n-1}(s ; q) \quad \text { and } \quad b(s ; q) \Phi_{n}(s ; q)=U_{n} \Phi_{n+1}(s ; q) .
$$

Again, without loss of generality, we will change the condition $[a(s ; q), b(s ; q)]_{\varsigma}=I$ into

$$
[a(s ; q), b(s ; q)]_{\varsigma}=\Lambda \text { and chose } \Lambda=\lambda_{1} .
$$

Also the operators $b(s ; q)=\mathfrak{a}_{\alpha}^{\uparrow}(s ; q)$ and $a(s ; q)=\mathfrak{a}_{\alpha}^{\downarrow}(s ; q)$, given in (12), provide the factorization of $\mathfrak{H}(s ; q)$.

If we now apply $b(s ; q)$ to the first equation of (26) and use the second one as well as (11), we find that $\lambda_{n}=D_{n} U_{n-1}$. On the other hand, applying $a(s ; q)$ to the second equation in (26) and using the first one as well as (15) we obtain $\lambda_{1}+\varsigma \lambda_{n}=U_{n} D_{n+1}=\lambda_{n+1}$. Thus, using Proposition 2.5 we conclude that $\lambda_{n}$ should be a $\varsigma$-linear function, i.e., $\lambda_{n}$ has the form $\lambda_{n}=A \varsigma^{n}+D$, where $A$ and $D$ are non-vanishing constants. Moreover, using the recurrence $\lambda_{1}+\varsigma \lambda_{n}=\lambda_{n+1}$ and Proposition 3.2 we obtain that

$$
\begin{aligned}
\mathfrak{H}(s ; q)\left\{a(s ; q) \Phi_{n}(s ; q)\right\} & =\lambda_{n-1}\left\{a(s ; q) \Phi_{n}(s ; q)\right\}, \\
\mathfrak{H}(s ; q)\left\{b(s ; q) \Phi_{n}(s ; q)\right\} & =\lambda_{n+1}\left\{b(s ; q) \Phi_{n}(s ; q)\right\},
\end{aligned}
$$


i.e., $a(s ; q) \Phi_{n}(s ; q)$ is the eigenvector associated to the eigenvalue $\lambda_{n-1}$ and $b(s ; q) \Phi_{n}(s ; q)$ is the eigenvector associated to the eigenvalue $\lambda_{n+1}$, so they really are the lowering and raising operators associated to the Hamiltonian $\mathfrak{H}(s ; q)$.

The next important question to be considered is when the $\alpha$-operators are mutually adjoint? Obviously the answer to this question depends on how the scalar product is defined. As an example, let us consider the discrete orthogonality case (8) and compute $\left\langle\mathfrak{a}_{\alpha}^{\downarrow} \Phi_{n+1}, \Phi_{k}\right\rangle$ :

$$
\begin{aligned}
& \left\langle\mathfrak{a}_{\alpha}^{\downarrow}(s ; q) \Phi_{n+1}(s ; q), \Phi_{k}(s ; q)\right\rangle= \\
= & \sum_{s=a}^{b-1} \frac{\sqrt{\nabla x_{1}(s) \rho(s)}}{d_{n} d_{n+1}}\left[e^{-\alpha \partial_{s}}\left(\sqrt{\frac{\sigma(s+1) \rho(s+1)}{\nabla x(s+1)}} P_{n+1}(s+1 ; q)-\sqrt{\frac{\sigma(-s-\mu) \rho(s)}{\Delta x(s)}} P_{n+1}(s ; q)\right)\right] P_{k}(s ; q) \\
= & \sum_{s=a}^{b-1} \frac{\sqrt{\nabla x_{1}(s) \rho(s)}}{d_{n} d_{n+1}}\left[e^{-\alpha \partial_{s}}\left(\sqrt{\frac{\sigma(-s-\mu) \rho(s)}{\Delta x(s)}} \Delta P_{n+1}(s ; q)\right)\right] P_{k}(s ; q) \\
= & \sum_{s=a}^{b-1} \frac{\sqrt{\nabla x_{1}(s) \rho(s)}}{d_{n} d_{n+1}} \sqrt{\frac{\sigma(-s-\mu+\alpha) \rho(s-\alpha)}{\Delta x(s-\alpha)}} \Delta P_{n+1}(s-\alpha ; q) P_{k}(s ; q),
\end{aligned}
$$

where the second equality follows from the Pearson equation (6). Next we have

$$
\begin{aligned}
\left\langle\Phi_{n+1}(s ; q), \mathfrak{a}_{\alpha}^{\uparrow}(s) \Phi_{k}(s ; q)\right\rangle= & \underbrace{\sum_{s=a}^{b-1} \frac{\sqrt{\sigma(s) \rho(s)}}{d_{n} d_{n+1} \sqrt{\nabla x(s)}}\left[e^{-\partial_{s}}\left(e^{\alpha \partial_{s}} \sqrt{\nabla x_{1}(s) \rho(s)} P_{k}(s ; q)\right)\right] P_{n+1}(s ; q)}_{S_{1}} \\
& -\underbrace{\sum_{s=a}^{b-1} \frac{\sqrt{\sigma(-s-\mu) \rho(s)}}{d_{n} d_{n+1} \sqrt{\Delta x(s)}}\left[e^{\alpha \partial_{s}}\left(\sqrt{\nabla x_{1}(s) \rho(s)} P_{k}(s ; q)\right)\right] P_{n+1}(s ; q)}_{S_{2}}
\end{aligned}
$$

If we use the boundary conditions $\left.\sigma(s) \rho(s)\right|_{s=a, b}=0$, then the sum $S_{1}$ becomes

$$
\begin{aligned}
S_{1} & =\sum_{s=a+1}^{b} \frac{\sqrt{\sigma(s) \rho(s)}}{d_{n} d_{n+1} \sqrt{\nabla x(s)}}\left[e^{-\partial_{s}}\left(e^{\alpha \partial_{s}} \sqrt{\nabla x_{1}(s) \rho(s)} P_{k}(s ; q)\right)\right] P_{n+1}(s ; q) \quad(s \rightarrow s+1) \\
& =\sum_{s=a}^{b-1} \frac{\sqrt{\sigma(s+1) \rho(s+1)}}{d_{n} d_{n+1} \sqrt{\nabla x(s+1)}}\left[\left(e^{\alpha \partial_{s}} \sqrt{\nabla x_{1}(s) \rho(s)} P_{k}(s ; q)\right)\right] P_{n+1}(s+1 ; q) \\
& =\sum_{s=a}^{b-1} \frac{\sqrt{\sigma(-s-\mu) \rho(s)}}{d_{n} d_{n+1} \sqrt{\Delta x(s)}}\left[e^{\alpha \partial_{s}}\left(\sqrt{\nabla x_{1}(s) \rho(s)} P_{k}(s ; q)\right)\right] P_{n+1}(s+1 ; q),
\end{aligned}
$$

where the last equality holds due to the Pearson equation (6). Now subtracting $S_{1}-S_{2}$ yields

$$
\begin{aligned}
\left\langle\Phi_{n+1}(s ; q), \mathfrak{a}_{\alpha}^{\uparrow}(s) \Phi_{k}(s ; q)\right\rangle & =\sum_{s=a}^{b-1} \frac{\sqrt{\sigma(-s-\mu) \rho(s)}}{d_{n} d_{n+1} \sqrt{\Delta x(s)}}\left[e^{\alpha \partial_{s}}\left(\sqrt{\nabla x_{1}(s) \rho(s)} P_{k}(s ; q)\right)\right] \Delta P_{n+1}(s ; q) \\
= & \sum_{s=a}^{b-1} \frac{\sqrt{\sigma(-s-\mu) \rho(s)}}{d_{n} d_{n+1} \sqrt{\Delta x(s)}} \sqrt{\nabla x_{1}(s+\alpha) \rho(s+\alpha)} P_{k}(s+\alpha ; q) \Delta P_{n+1}(s ; q) .
\end{aligned}
$$


Then, in the discrete case, a sufficient condition for the operators $\mathfrak{a}_{\alpha}^{\uparrow}(s ; q)$ and $\mathfrak{a}_{\alpha}^{\downarrow}(s ; q)$ to be mutually adjoint, i.e., $\left\langle\mathfrak{a}_{\alpha}^{\downarrow}(s) \Phi_{n+1}(s ; q), \Phi_{n}(s ; q)\right\rangle=\left\langle\Phi_{n+1}(s ; q), \mathfrak{a}_{\alpha}^{\uparrow}(s) \Phi_{k}(s ; q)\right\rangle$, is that $\alpha=0$. For the general case as well as for the discrete cases when $\alpha \neq 0$, the problem requires a more detailed study of each case. A discussion of these cases will be considered elsewhere.

\section{Examples}

In this section we will exhibit several examples involving some well-known families of $q$-polynomials. Let us point out that from the whole $q$-Askey tableau [7] we need only to consider those cases, when $\lambda_{n}$ is a $q$-linear or $q^{-1}$-linear function of $n$ (see Theorem 3.5).

\subsection{The linear lattice $x(s)=c_{1} q^{s}+c_{3}$ : The $q$-Hahn Tableau}

We start with the so-called $q$-Hahn tableau (see e.g. $[8,7]$ and references therein). Taking into account 3.4 , the $\alpha$-operators are chosen in such a way that $A(s)=\sqrt{\nabla x_{1}(s)}$, so the orthonormal functions are defined by

$$
\Phi_{n}(s ; q)=d_{n}{ }^{-1} \sqrt{\rho(s) \nabla x_{1}(s)} P_{n}(s ; q)
$$

and satisfy the discrete orthogonality relation

$$
\sum_{s_{i}=a}^{b-1} \Phi_{n}\left(s_{i} ; q\right) \Phi_{m}\left(s_{i} ; q\right)=\delta_{n m} .
$$

By using the theorem 3.4, one can solve the factorization problem 1 for the $q$-Hahn tableau.

Before starting with some relevant examples, we recall that for the $q$-linear lattice $x(s)=$ $c_{1} q^{s}+c_{3}$,

$$
\mathfrak{e}_{q}(s):=\frac{\nabla x(s)}{\nabla x_{1}(s-\alpha)} \sqrt{\frac{\nabla x_{1}(s-1) \nabla x_{1}(s)}{\nabla x(s-\alpha) \Delta x(s-\alpha)}}=q^{2 \alpha-1},
$$

whereas if $x(s)=c_{1} q^{-s}+c_{3}$, then $\mathfrak{e}_{q}(s)=q^{-2 \alpha+1}$.

Remark 4.1 Let $x(s)=q^{s}$. Then in the case when $\sigma(s)$ or $\sigma(-s-\mu)=\sigma(s)+\tau(s) \nabla x_{1}(s)$ are constants, the operators (12) define a dynamical algebra if and only if $\alpha=1$ and $\varsigma=q$ or $\alpha=0$ and $\varsigma=q^{-1}$, respectively. To prove this assertion it is sufficient to use the formula (18) that yields, for the first case

$$
\mathfrak{e}_{q}(s) \sqrt{\frac{\sigma(-s-\mu+\alpha)}{\sigma(-s-\mu+1)}}=q^{2 \alpha-1} \sqrt{\frac{\sigma(-s-\mu+\alpha)}{\sigma(-s-\mu+1)}}=\varsigma .
$$

Choosing $\alpha=1$ we have that $\varsigma=q$. The other case is analogous. These cases constitute the most simple ones.

\subsubsection{Stieltjes-Wigert $q$-polynomials $S_{n}(x ; q)$}

The Stieltjes-Wigert functions in $x(s)=q^{s}$, i.e. the functions, associated with the StieltjesWigert polynomials, are defined by

$$
\begin{gathered}
\Phi_{n}(x ; q)=\frac{1}{d_{n}} \sqrt{(-x,-q / x ; q)_{\infty}} 1 \phi_{1}\left(\begin{array}{c}
q^{-n} \\
0
\end{array} \mid q ;-x q^{n+1}\right), \quad x(s)=q^{s}, \\
d_{n}=\frac{q^{n / 2}}{(q ; q)_{\infty}} \sqrt{\frac{\left(q^{n+1} ; q\right)_{\infty}}{\log q^{-1}}} .
\end{gathered}
$$


In this case we have chosen $A(s)=\sqrt{\nabla x_{1}(s)}$. The above functions $\Phi_{n}(x ; q)$ possess the following orthogonality property $\int_{0}^{\infty} \Phi_{n}(x ; q) \Phi_{m}(x ; q) d x=\delta_{n, m}$.

Since for the Stieltjes-Wigert $q$-polynomials $\sigma(s)=q^{s-1}$ and $\sigma(s)+\tau(s) \nabla x_{1}(s)=q^{2 s}$ [7], one can define the following Hamiltonian

$$
\mathfrak{H}(s ; q)=\frac{1}{(1-q)}\left\{\left(1+q^{-s}\right) I-q^{-(s+1) / 2} e^{\partial_{s}}-q^{-s / 2} e^{-\partial_{s}}\right\},
$$

for which one has $\mathfrak{H}(s ; q) \Phi_{n}\left(q^{s} ; q\right)=\frac{1-q^{n}}{1-q} \Phi_{n}\left(q^{s} ; q\right)$.

Next we check the conditions of the Theorem 3.4: The first condition (18) yields $\varsigma=q^{\alpha / 2}$ and substituting it in the second one, we obtain that it holds when $\alpha=2$, i.e., $\varsigma=q$ and therefore

$$
\begin{aligned}
& \mathfrak{a}^{\downarrow}(s ; q):=\mathfrak{a}_{2}^{\downarrow}(s ; q)=\frac{1}{\sqrt{1-q}}\left(e^{-2 \partial_{s}}-e^{-\partial_{s}} q^{-s / 2}\right), \\
& \mathfrak{a}^{\uparrow}(s ; q):=\mathfrak{a}_{2}^{\uparrow}(s ; q)=\frac{1}{\sqrt{1-q}}\left(e^{2 \partial_{s}}-q^{-s / 2} e^{\partial_{s}}\right),
\end{aligned}
$$

$\mathfrak{H}(s ; q)=\mathfrak{a}^{\uparrow}(s ; q) \mathfrak{a}^{\downarrow}(s ; q)$ and $\left[\mathfrak{a}^{\downarrow}(s ; q), \mathfrak{a}^{\uparrow}(s ; q)\right]_{q}=I$. It is not hard to verify that in this case the operators $\mathfrak{a}^{\uparrow}(s ; q)$ and $\mathfrak{a}^{\downarrow}(s ; q)$ are the lowering and raising operators for the functions $\Phi_{n}\left(q^{s} ; q\right)$ (see [7], p.117, (3.27.6) and (3.27.8)). We remind the reader that the moment problem, associated with the Stieltjes-Wigert polynomials, is indeterminate $[22,23]$ and therefore there are several distinct weight functions (both continuous and discrete ones), with respect to which they are orthogonal. A similar result for the case of a discrete orthogonality condition has been first considered in [12].

\subsubsection{Al-Salam \& Carlitz I and II $q$-polynomials $U_{n}^{(a)}(x ; q)$ and $V_{n}^{(a)}(x ; q)$}

The Al-Salam-Carlitz polynomials of type I and of type II, depend on an additional parameter $a$ and therefore they occupy the next level in the Askey scheme (see [7, p.114]). Since these two families are interrelated,

$$
V_{n}^{(a)}(x ; q)=U_{n}^{(a)}\left(x ; q^{-1}\right),
$$

it is sufficient to consider only one of them.

Let us define the functions $[7$, p. 114]

$$
\begin{gathered}
\Phi_{n}(s ; q)=\frac{1}{d_{n}} \frac{a^{s / 2} q^{s^{2} / 2}}{\sqrt{(q, a q ; q)_{s}}}{ }_{2} \phi_{0}\left(\begin{array}{c}
q^{-n}, q^{-s} \mid q ; \frac{q^{n}}{a} \\
-
\end{array}\right), \quad a>0 \\
d_{n}=(-1)^{n}(a q)^{-n / 2} \sqrt{\frac{(q ; q)_{n}}{(a q ; q)_{\infty}}}
\end{gathered}
$$

which satisfy the discrete orthogonality relation $\sum_{k=0}^{\infty} \Phi_{n}(k ; q) \Phi_{m}(k ; q)=\delta_{m, n}$. Since for this family $\sigma(s)=\left(q^{-s}-1\right)\left(q^{-s}-a\right)$ and $\sigma(s)+\tau(s) \nabla x_{1}(s)=a$, where $x(s)=q^{-s}$, then one derives that the difference Hamiltonian $\mathfrak{H}(s ; q)$ has the following form

$$
\begin{aligned}
\mathfrak{H}(s ; q)= & \frac{1}{1-q}\left[a q^{2 s+1}+\left(1-q^{s}\right)\left(1-a q^{s}\right)\right. \\
& \left.-e^{\partial_{s}} \sqrt{a\left(1-q^{s}\right)\left(1-a q^{s}\right)} q^{s-1 / 2}-\sqrt{a\left(1-q^{s}\right)\left(1-a q^{s}\right)} q^{s-1 / 2} e^{-\partial_{s}}\right] .
\end{aligned}
$$

Thus $\mathfrak{H}(s ; q) \Phi_{n}(s ; q)=\frac{1-q^{n}}{1-q} \Phi_{n}(s ; q)$. This Hamiltonian is factorized, $\mathfrak{H}(s ; q)=\mathfrak{a}^{\uparrow}(s ; q) \mathfrak{a}^{\downarrow}(s ; q)$, in terms of the difference operators

$$
\mathfrak{a}^{\downarrow}(s ; q):=\mathfrak{a}_{0}^{\downarrow}(s ; q)=\frac{1}{\sqrt{1-q}}\left[\sqrt{a} q^{s+1 / 2}-e^{\partial_{s}} \sqrt{\left(1-q^{s}\right)\left(1-a q^{s}\right)}\right],
$$




$$
\mathfrak{a}^{\uparrow}(s ; q):=\mathfrak{a}_{0}^{\uparrow}(s ; q)=\frac{1}{\sqrt{1-q}}\left[\sqrt{a} q^{s+1 / 2}-\sqrt{\left(1-q^{s}\right)\left(1-a q^{s}\right)} e^{-\partial_{s}}\right],
$$

that satisfy the commutation relation $\left[\mathfrak{a}^{\downarrow}(s ; q), \mathfrak{a}^{\uparrow}(s ; q)\right]_{q}=I$. Thus the dynamical algebra for this family is also $s u_{q}(1,1)$. As in the previous case, the operators $\mathfrak{a}_{2}^{\downarrow}(s ; q)$ and $\mathfrak{a}^{\uparrow}(s ; q)$ are the lowering and raising operators for the functions $\Phi_{n}(s ; q)$ (see [7]).

A special case of the Al-Salam\&Carlitz $q$-polynomials of type II are the discrete Hermite $q$-polynomials $\widetilde{h}_{n}(x ; q)=\mathrm{i}^{-n} V_{n}^{(-1)}(\mathrm{i} x ; q)$ of type II.

All these cases are closely related to some models of $q$-oscillators $[10,12,13,14,15,16]$.

\subsubsection{Wall polynomials $p_{n}(x ; a \mid q)$}

Our next example is the little $q$-Laguerre / Wall polynomials. In this case we define the function

$$
\Phi_{n}(s ; a ; q)=\frac{1}{d_{n}(a ; q)} \frac{(a q)^{s / 2}}{\sqrt{(q ; q)_{s}}}{ }_{2} \phi_{1}\left(\begin{array}{c}
q^{-n}, 0 \\
a q
\end{array} \mid q ; q^{s+1}\right),
$$

where $x:=x(s)=q^{s}$ and $A(s)=\sqrt{\nabla x_{1}(s)}$.

This function satisfies the discrete orthogonality relation $\sum_{k=0}^{\infty} \Phi_{n}(k ; q) \Phi_{m}(k ; q)=\delta_{n, m}$, provided that

$$
d_{n}(a ; q)=\frac{(a q)^{n / 2}}{(a q ; q)_{\infty}} \sqrt{(q ; q)_{n}\left(a q^{n+1} ; q\right)_{\infty}} .
$$

In this case $\sigma(s)=q^{-1} q^{s}\left(q^{s}-1\right)$ and $\sigma(s)+\tau(s) \nabla x_{1}(s)=-a q^{s}$, thus the corresponding Hamiltonian is

$$
\mathfrak{H}(s ; a ; q)=\frac{1}{(1-q) x}\left(q(a+1-x) I-\sqrt{a q(1-q x)} e^{\partial_{s}}-q \sqrt{a q(1-x)} e^{-\partial_{s}}\right)
$$

and $\mathfrak{H}(s ; a ; q) \Phi_{n}(s ; a ; q)=\frac{1-q^{-n}}{1-q^{-1}} \Phi_{n}(s ; a ; q)$.

Then, the first condition of Theorem 3.4 leads to the value $\alpha=0$ and $\varsigma=q^{-1 / 2}$ and the second condition holds if and only if the parameter $a$ of the above functions is equal to $q^{-1 / 2}$, but they do not lead to the lowering and raising operators.

So to introduce the lowering and raising operators one has to consider the following operators

$$
\begin{aligned}
& \mathfrak{a}(s ; a ; q):=\frac{1}{\sqrt{(1-q) x}}\left(\sqrt{(1-q x)} e^{\partial_{s}}-\sqrt{a q} I\right), \\
& \mathfrak{a}^{\dagger}(s ; a ; q):=\frac{1}{\sqrt{(1-q) x}}\left(\sqrt{q(1-x)} e^{-\partial_{s}}-\sqrt{a q} I\right) .
\end{aligned}
$$

The above mutually adjoint operators factorize the Hamiltonian $\mathfrak{H}(s ; a ; q)$, i.e., $\mathfrak{H}(s ; a ; q)=$ $\mathfrak{a}^{\dagger}(s ; a ; q) \mathfrak{a}(s ; a ; q)$, and they satisfy the commutation relation

$$
\mathfrak{a}(s ; a / q ; q) \mathfrak{a}^{\dagger}(s ; a / q ; q)-q^{-1} \mathfrak{a}^{\dagger}(s ; a ; q) \mathfrak{a}(s ; a ; q)=I .
$$

From this relation it follows that their action on the functions $\Phi(s ; a ; q)$ is given by

$$
\begin{aligned}
& \mathfrak{a}(s ; a ; q) \Phi_{n}(s ; a ; q)=\sqrt{\frac{1-q^{-n}}{1-q^{-1}}} \Phi_{n-1}(s ; a q ; q), \\
& \mathfrak{a}^{\dagger}(s ; a / q ; q) \Phi_{n}(s ; a ; q)=\sqrt{\frac{1-q^{-n-1}}{1-q^{-1}}} \Phi_{n+1}(s ; a / q ; q) .
\end{aligned}
$$


To verify these formulae one needs to use the property of the normalization constant $d_{n}(a ; q)$ that

$$
d_{n}(a ; q)=\frac{(1-a) q^{n / 2}}{\sqrt{a\left(1-q^{n+1}\right)}} d_{n+1}(a / q ; q) .
$$

Observe that the operators $\mathfrak{a}(s ; a ; q)$ and $\mathfrak{a}^{\dagger}(s ; a ; q)$ not only lower and raise, respectively, the index $n$, but they alter also the parameter $a$. That is why an appropriate dynamical algebra in this case is not $s u_{q}(1,1)$ (see the discussion above, which follows the proof of Theorem 3.4 in section 3). The formulae (27) are equivalent to proving that the forward and backward shift operators for the little $q$-Laguerre polynomials have the form (see [7] p. 107, (3.20.6) and $(3.20 .8))$

$$
\begin{gathered}
\left(e^{\partial_{s}}-I\right) p_{n}\left(q^{s} ; a ; q\right)=q^{s+1-n} \frac{1-q^{n}}{1-a q} p_{n-1}\left(q^{s} ; a q ; q\right), \\
{\left[\left(1-q^{s}\right) e^{-\partial_{s}}-a I\right] p_{n}\left(q^{s} ; a ; q\right)=(1-a) p_{n+1}\left(q^{s} ; a / q ; q\right),}
\end{gathered}
$$

respectively.

To conclude this example let us mention that the Charlier $q$-polynomials $c_{n}^{(\mu)}(s ; q)$ on the lattice $x(s)=q^{s}$, introduced in [24], are a special case of the Wall polynomials, considered above.

\subsubsection{Discrete Laguerre $q$-polynomials $L_{n}^{(\alpha)}(x ; q)$}

Let us consider now the Hamiltonian, associated with the discrete Laguerre $q$-polynomials. For these polynomials $\sigma(s)=q^{-1} q^{s}$ and $\sigma(s)+\tau(s) \nabla x_{1}(s)=a q^{s}\left(q^{s}+1\right)$ [7]. If we put $A(s)=$ $\sqrt{\nabla x_{1}(s)}$ and use the condition (18), then we obtain that $\alpha=1$ and $\varsigma=\sqrt{q}$. Next we use the second condition (19), but the corresponding expression is a constant if and only if $a=q^{-1 / 2}$, i.e., not for any value of the parameter $a$ the $q$-Hamiltonian, associated with the discrete Laguerre $q$-polynomials, can be factorized by using the $\alpha$-operators, which satisfy the corresponding commutation relation. But as in the previous case, one can define the functions

$$
\Phi_{n}^{(\alpha)}(s ; q)=d_{n}^{-1}(\alpha) \frac{q^{s / 2(\alpha+1)}}{\sqrt{\left(-q^{s} ; q\right)_{\infty}}} L_{n}^{(\alpha)}(x ; q), \quad x:=x(s)=q^{s},
$$

where the normalization constant $d_{n}(\alpha)$ is given by

$$
d_{n}(\alpha):=\frac{\left(-q^{\alpha+1},-q^{\alpha} ; q\right)_{\infty}^{1 / 2}}{(q ; q)_{\infty}} \frac{(q ; q)_{n+\alpha}^{1 / 2}}{q^{n / 2}(q ; q)_{n}^{1 / 2}},
$$

and the discrete Laguerre $q$-polynomials $L_{n}^{(\alpha)}(x ; q)$ are

$$
L_{n}^{(\alpha)}(x ; q):=\frac{1}{(q ; q)_{n}}{ }_{2} \phi_{1}\left(\begin{array}{c|c}
q^{-n}, x \\
0
\end{array} \mid q ; q^{n+\alpha+1}\right) .
$$

These functions satisfy the discrete orthogonality relation $\sum_{k=0}^{\infty} \Phi_{n}^{(\alpha)}(k ; q) \Phi_{m}^{(\alpha)}(k ; q)=\delta_{n, m}$ and

$$
\mathfrak{H}^{(\alpha)}(s ; q) \Phi_{n}^{(\alpha)}(s ; q)=\frac{1-q^{n}}{1-q} \Phi_{n}^{(\alpha)}(s ; q),
$$

where the $q$-Hamiltonian is a difference operator of the form

$$
\mathfrak{H}^{(\alpha)}(s ; q)=\frac{1}{1-q}\left\{\left[1+\left(1+q^{-\alpha}\right) q^{-s}\right] I-q^{-\frac{s+\alpha}{2}}\left[\sqrt{1+q^{s}} e^{\partial_{s}}+e^{-\partial_{s}} \sqrt{1+q^{s}}\right] q^{-\frac{s}{2}}\right\} .
$$


In this case the mutually adjoint operators, that factorize the above $q$-Hamiltonian, are

$$
\begin{aligned}
& \mathfrak{a}(s ; \alpha ; q):=\frac{1}{\sqrt{1-q}}\left(q^{-\frac{\alpha}{2}} I-e^{-\partial_{s}} \sqrt{1+q^{s}}\right) q^{-\frac{s}{2}}, \\
& \mathfrak{a}^{\dagger}(s ; \alpha ; q):=\frac{1}{\sqrt{1-q}} q^{-\frac{s}{2}}\left(q^{-\frac{\alpha}{2}} I-\sqrt{1+q^{s}} e^{\partial_{s}}\right) .
\end{aligned}
$$

They satisfy the commutation relation

$$
\mathfrak{a}(s ; \alpha-1 ; q) \mathfrak{a}^{\dagger}(s ; \alpha-1 ; q)-q \mathfrak{a}^{\dagger}(s ; \alpha ; q) \mathfrak{a}(s ; \alpha ; q)=I,
$$

from which it follows that

$$
\begin{aligned}
& \mathfrak{a}(s ; \alpha ; q) \Phi_{n}^{(\alpha)}(s ; q)=\sqrt{\frac{1-q^{n}}{1-q}} \Phi_{n-1}^{(\alpha+1)}(s ; q), \\
& \mathfrak{a}^{\dagger}(s ; \alpha-1 ; q) \Phi_{n}^{(\alpha)}(s ; q)=\sqrt{\frac{1-q^{n+1}}{1-q}} \Phi_{n+1}^{(\alpha-1)}(s ; q) .
\end{aligned}
$$

As is the previous case, these relations yield precisely the explicit form of the forward and backward shift operators, respectively, for the $q$-Laguerre polynomials $L_{n}^{(\alpha)}\left(q^{s} ; q\right)$ (see [7], p. $109,(3.21 .7)$ and $(3.21,9))$.

\subsubsection{Other cases in the $q$-Hahn tableau}

If one now applies the theorems 3.5 and 3.4 to the families of big $q$-Jacobi polynomials with $b=0$ (big $q$-Laguerre), little $q$-Jacobi, $q$-Meixner, $q$-Kravchuck, quantum $q$-Kravchuk, affine $q$-Kravchuk and alternative $q$-Charlier polynomials, then for all these cases it is impossible to solve the problem 1 . The big and little $q$-Jacobi polynomials do not admit a dynamical algebra because the corresponding eigenvalues are not $q$-linear functions of $n$ and in the other cases one of the two conditions of theorem 3.4 fails.

As an example let us consider the case of the $q$-Meixner polynomials. For the $q$-Meixner polynomials [7], we have $x(s)=q^{-s}, \sigma(s)=c(x(s)-b q) / q, \sigma(s)+\tau(s) \nabla x_{1}(s)=(x(s)-$ 1) $(x(s)+b c)$ and $\lambda_{n}=q^{1 / 2} \frac{1-q^{n}}{(1-q)^{2}}$. Then, the condition (18) gives

$$
q^{\frac{1}{2}-\alpha} \frac{q^{-2 \alpha+1}\left(q^{\alpha}-q^{s}\right)\left(b c q^{s}+q^{\alpha}\right)\left(q^{\alpha}-b q^{s+1}\right)}{\left(q^{s}-q\right)\left(b c q^{s}+q\right)\left(b q^{s+1}-1\right)}=\varsigma .
$$

After a careful study of the left-hand side one arrives at the conclusion that it is constant if and only if $\alpha=2 / 3, b=q^{-4 / 3}, c=-q^{5 / 3}$ or $\alpha=2 / 3, b=q^{-5 / 3}, c=-q^{4 / 3}$. After substituting this in the second condition (19), it becomes clear that the only possibility is the first one, but it corresponds to a non-positive case.

Let us consider this case in more details.

The $q$-Meixner polynomials ([7], p.95),

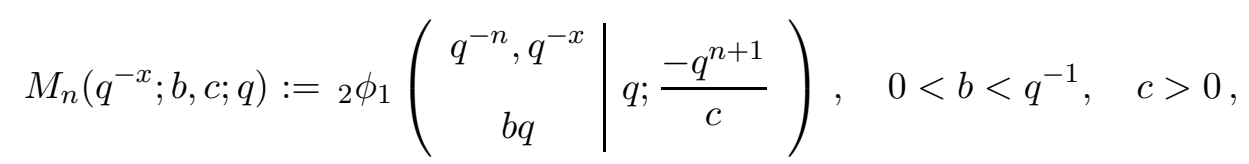

depend on two parameters $b$ and $c$ (in addition to the base $q$ ) and occupy one level higher in the Askey scheme than $q$-Charlier and Al-Salam-Carlitz polynomials of type II. They satisfy a difference equation

$$
\left[B(x)\left(1-e^{\partial_{x}}\right)+D(x)\left(1-e^{-\partial_{x}}\right)\right] M_{n}\left(q^{-x} ; b, c ; q\right)=\left(1-q^{n}\right) M_{n}\left(q^{-x} ; b, c ; q\right),
$$




$$
B(x)=c q^{x}\left(1-b q^{x+1}\right), \quad D(x)=\left(1-q^{x}\right)\left(1+b c q^{x}\right) .
$$

So $q$-Meixner functions, defined as

$$
\Phi_{n}^{M}(x ; b, c ; q):=d_{n}^{-1}(b, c)\left[\frac{c^{x}(b q ; q)_{x}}{(q ; q)_{x}(-b c q ; q)_{x}}\right]^{1 / 2} q^{x(x-1) / 4} M_{n}\left(q^{-x} ; b, c ; q\right),
$$

are eigenfunctions of a difference "Hamiltonian" $\mathfrak{H}^{M}(x ; b, c ; q)$,

$$
\mathfrak{H}^{M}(x ; b, c ; q) \Phi_{n}^{M}(x ; b, c ; q)=\frac{1-q^{n}}{1-q} \Phi_{n}^{M}(x ; b, c ; q),
$$

where

$$
\mathfrak{H}^{M}(x ; b, c ; q):=\frac{1}{1-q}\left[B(x)+D(x)-B^{1 / 2}(x) e^{\partial_{x}} D^{1 / 2}(x)-D^{1 / 2}(x) e^{-\partial_{x}} B^{1 / 2}(x)\right] .
$$

The $q$-Meixner functions satisfy the discrete orthogonality relation

$$
\sum_{k=0}^{\infty} \Phi_{m}^{M}(k ; b, c ; q) \Phi_{n}^{M}(k ; b, c ; q)=\delta_{m n} .
$$

One can factorize the "Hamiltonian" $\mathfrak{H}^{M}(x ; b, c ; q)$,

$$
\mathfrak{H}^{M}(x ; b, c ; q)=a_{M}^{\uparrow}(x ; b, c ; q) a_{M}^{\downarrow}(x ; b, c ; q),
$$

by means of the "lowering" and "raising" difference operators

$$
\begin{aligned}
\mathfrak{a}_{M}^{\downarrow}(x ; b, c ; q) & :=\frac{1}{\sqrt{1-q}}\left[e^{\partial_{x}} D^{1 / 2}(x)-B^{1 / 2}(x)\right], \\
\mathfrak{a}_{M}^{\uparrow}(x ; b, c ; q) & :=\frac{1}{\sqrt{1-q}}\left[D^{1 / 2}(x) e^{-\partial_{x}}-B^{1 / 2}(x)\right] .
\end{aligned}
$$

The difference operators (29) satisfy a $q$-commutation relation of the form

$$
\mathfrak{a}_{M}^{\downarrow}(x ; b / q, c q ; q) \mathfrak{a}_{M}^{\uparrow}(x ; b / q, c q ; q)-q \mathfrak{a}_{M}^{\uparrow}(x ; b, c ; q) \mathfrak{a}_{M}^{\downarrow}(x ; b, c ; q)=I .
$$

Their action on the $q$-Meixner functions is given by

$$
\begin{aligned}
& \mathfrak{a}_{M}^{\downarrow}(x ; b, c ; q) \Phi_{n}^{M}(x ; b, c ; q)=\sqrt{\frac{1-q^{n}}{1-q}} \Phi_{n-1}^{M}(x ; b q, c / q ; q), \\
& \mathfrak{a}_{M}^{\uparrow}(x ; b / q, c q ; q) \Phi_{n}^{M}(x ; b, c ; q)=\sqrt{\frac{1-q^{n+1}}{1-q}} \Phi_{n+1}^{M}(x ; b / q, c q ; q),
\end{aligned}
$$

that is, they not only lower and raise, respectively, the index $n$, but alter also the parameters $b$ and $c$. The formulae (30) are equivalent to the statement that the forward and backward shift operators for the $q$-Meixner polynomials (28) have the form (see [7], p.95, (3.13.2) and (3.13.8))

$$
\begin{gathered}
\left(1-e^{\partial_{x}}\right) M_{n}\left(q^{-x} ; b, c ; q\right)=\frac{1-q^{n}}{c(1-b q)} q^{-x} M_{n-1}\left(q^{-x} ; b q, c / q ; q\right), \\
{\left[c q^{x}\left(1-b q^{x}\right)-\left(1-q^{x}\right)\left(1+b c q^{x}\right) e^{-\partial_{x}}\right] M_{n}\left(q^{-x} ; b, c ; q\right)} \\
=c q^{x}(1-b) M_{n+1}\left(q^{-x} ; b / q, c q ; q\right) .
\end{gathered}
$$

It remains only to remind the reader that when the parameter $b$ in $(28)$ vanishes, the $q$ Meixner polynomials $M_{n}\left(q^{-x} ; 0, c ; q\right)$ coincide with the $q$-Charlier polynomials ([7], p.112)

$$
C_{n}\left(q^{-x} ; c ; q\right):={ }_{2} \phi_{1}\left(\begin{array}{c|c}
q^{-n}, q^{-x} & q ; \frac{-q^{n+1}}{c} \\
0 &
\end{array}\right) .
$$

In this case $B(x)=c q^{x}$ and $D(x)=1-q^{x}$. The appropriate formulae for the $q$-Charlier polynomials (31) are therefore easy consequences of the corresponding formulae for the $q$-Meixner polynomials (28) with the vanishing value of the parameter $b$. 


\subsection{Askey-Wilson cases}

Using the linearity of the difference equation (1) we can consider, with not loss of generality, the following lattice $x(s)=\frac{1}{2}\left(q^{s}+q^{-s}\right)$, for which $\mu=0$. Then,

$$
\sigma(s)=C q^{-2 s} \prod_{i=1}^{4}\left(q^{s}-q^{s_{i}}\right)=q^{-2 s} \prod_{i=1}^{4}\left(q^{s}-z_{i}\right), \quad \sigma(-s-\mu)=C q^{2 s} \prod_{i=1}^{4}\left(q^{-s}-z_{i}\right) .
$$

It is well known that the general case when the zeros of $\tilde{\sigma}$, namely $z_{1} z_{2} z_{3} z_{4} \neq 0$, corresponds to the Askey-Wilson polynomials [7]. We will use the theorem 3.4 to solve the factorization problem for the whole $q$-Askey tableau [7] and Nikiforov-Uvarov tableau $[4,5]$.

The Askey-Wilson polynomials on the lattice $x(s)=\frac{1}{2}\left(q^{s}+q^{-s}\right)=\cos \theta$ where $q^{s}=e^{i \theta}$, defined by $[7]$

$$
p_{n}(x(s) ; a, b, c, d \mid q)=\frac{(a b, a c, a d ; q)_{n}}{a^{n}}{ }_{4} \varphi_{3}\left(\begin{array}{c}
q^{-n}, q^{n-1} a b c d, a q^{-s}, a q^{s} \\
a b, a c, a d
\end{array} \mid q ; q\right),
$$

where $a=z_{1}, b=z_{2}, c=z_{3}, d=z_{4}$ and $\mu=0$. Their orthogonality relation is of the form

$$
\int_{-1}^{1} \omega(x) p_{n}(x ; a, b, c, d) p_{m}(x ; a, b, c, d) d x=\delta_{n m} d_{n}^{2}
$$

where

$$
\omega(x)=\frac{h(x, 1) h(x,-1) h\left(x, q^{\frac{1}{2}}\right) h\left(x,-q^{\frac{1}{2}}\right)}{2 \pi \sqrt{1-x^{2}} h(x, a) h(x, b) h(x, c) h(x, d)}, \quad h(x, \alpha)=\prod_{k=0}^{\infty}\left[1-2 \alpha x q^{k}+\alpha^{2} q^{2 k}\right],
$$

and the norm is given by

$$
d_{n}^{2}=\frac{\left(a b c d q^{n-1}, a b c d q^{2 n} ; q\right)_{\infty}}{\left(q^{n+1}, a b q^{n}, a c q^{n}, a d q^{n}, b c q^{n}, b d q^{n}, c d q^{n} ; q\right)_{\infty}} .
$$

The Askey-Wilson functions can be defined by

$$
\Phi_{n}(s ; q)=\sqrt{\frac{\omega(s) A^{2}(s)}{d_{n}^{2}}} p_{n}(x(s) ; a, b, c, d), \quad x(s)=\cos \theta, \quad q^{s}=e^{i \theta} .
$$

Taking $A(s)=\sqrt{\nabla x_{1}(s)}$, we have the orthogonality $\int_{-1}^{1} \Phi_{n}(s ; q) \Phi_{m}(s ; q) / \nabla x_{1}(s) d x=\delta_{n, m}$, and $\mathfrak{H}(s ; q) \Phi_{n}(s ; q)=\lambda_{n} \Phi_{n}(s ; q)$, where $\lambda_{n}=q\left(q^{-n}-1\right)\left(1-a b c d q^{n-1}\right)$, and $\mathfrak{H}(s ; q)$ is given by $(9), \sigma(s)=C_{\sigma} q^{-2 s}\left(q^{s}-a\right)\left(q^{s}-b\right)\left(q^{s}-c\right)\left(q^{s}-d\right)$ and $\mu=0$. Thus the Hamiltonian, associated with these Askey-Wilson functions, is

$$
\begin{gathered}
\mathfrak{H}(s ; q)=\frac{-1}{k_{q}^{2} \sqrt{\sin \theta}}\left(\frac{\sqrt{\sigma(s) \sigma(-s+1)}}{\sin \left(\theta+\frac{i}{2} \log q\right) \sqrt{\sin (\theta+i \log q)}} e^{-\partial_{s}}+\frac{\sqrt{\sigma(s+1) \sigma(-s)}}{\sin \left(\theta-\frac{i}{2} \log q\right) \sqrt{\sin (\theta-i \log q)}} e^{\partial_{s}}\right) \\
\quad+\frac{1}{k_{q}^{2} \sin \theta}\left(\frac{\sigma(s)}{\sin \left(\theta+\frac{i}{2} \log q\right)}+\frac{\sigma(-s)}{\sin \left(\theta-\frac{i}{2} \log q\right)}\right) I .
\end{gathered}
$$

If we now use the Remark 3.6, then the first condition of the Theorem 3.4 holds for $\alpha=\frac{1}{2}$. In fact, a straightforward calculations shows that

$$
\Delta x_{1}(s+\gamma)=\frac{k_{q}}{2} q^{-s-\gamma}\left(q^{s+\gamma}+1\right)\left(q^{s+\gamma}-1\right) .
$$


Hence, the condition (18) has the form $\left(e^{i \theta}=q^{s}\right)$

$$
\begin{gathered}
q^{-2 \alpha+1}\left(\frac{q^{s-\frac{1}{2}}-1}{q^{s-\alpha}-1} \sqrt{\frac{\left(q^{s-1}-1\right)\left(q^{s}-1\right)}{\left(q^{s-\alpha-\frac{1}{2}}-1\right)\left(q^{s-\alpha+\frac{1}{2}}-1\right)}} \sqrt{\prod_{i=1}^{4} \frac{\left(q^{s-\alpha}-z_{i}\right)\left(q^{-s+\alpha}-z_{i}\right)}{\left(q^{s}-z_{i}\right)\left(q^{-s+1}-z_{i}\right)}}\right) \times \\
\frac{q^{s-\frac{1}{2}}+1}{q^{s-\alpha}+1} \sqrt{\frac{\left(q^{s-1}+1\right)\left(q^{s}+1\right)}{\left(q^{s-\alpha-\frac{1}{2}}+1\right)\left(q^{s-\alpha+\frac{1}{2}}+1\right)}}=\varsigma .
\end{gathered}
$$

If we look at the expression in the second line, we find that it is a constant if and only if $\alpha=\frac{1}{2}$, and thus the first condition transforms into

$$
\sqrt{\frac{\prod_{i=1}^{4}\left(q^{s-1 / 2}-z_{i}\right)\left(q^{-s+1 / 2}-z_{i}\right)}{\prod_{i=1}^{4}\left(q^{s}-z_{i}\right)\left(q^{-s+1}-z_{i}\right)}}=\varsigma .
$$

Then, the simplest case for which the condition (18) holds is when $z_{1} z_{2}=q^{\frac{1}{2}}$ and $z_{3} z_{4}=q^{\frac{1}{2}}$ (with the corresponding permutations of the roots). In this case $\varsigma=1$. Since $\sigma(s)=\sigma\left(-s+\frac{1}{2}\right)$, the second condition gives

$$
\frac{1}{\nabla x_{1}(s)}\left(\frac{\sigma\left(s+\frac{1}{2}\right)}{\Delta x(s)}+\frac{\sigma\left(-s+\frac{1}{2}\right)}{\nabla x(s)}-\frac{\sigma(s)}{\nabla x(s)}-\frac{\sigma(-s)}{\Delta x(s)}\right)=0 .
$$

Thus, we have $a_{1 / 2}^{\uparrow}(s ; q) a_{1 / 2}^{\downarrow}(s)=\mathfrak{H}(s ; q)$ and $\left[a_{1 / 2}^{\downarrow}(s ; q), a_{1 / 2}^{\uparrow}(s)\right]_{q}=0$, where

$$
\begin{aligned}
& a_{1 / 2}^{\downarrow}(s ; q)=e^{\frac{1}{2} \partial_{s}} \sqrt{\frac{\sigma(s)}{-k_{q}^{2} \sin \theta \sin \left(\theta+\frac{i}{2} \log q\right)}}-e^{-\frac{1}{2} \partial_{s}} \sqrt{\frac{\sigma(-s)}{-k_{q}^{2} \sin \theta \sin \left(\theta-\frac{i}{2} \log q\right)}}, \\
& a_{1 / 2}^{\uparrow}(s ; q)=\sqrt{\frac{\sigma(s)}{-k_{q}^{2} \sin \theta \sin \left(\theta+\frac{i}{2} \log q\right)}} e^{-\frac{1}{2} \partial_{s}}-\sqrt{\frac{\sigma(-s)}{-k_{q}^{2} \sin \theta \sin \left(\theta-\frac{i}{2} \log q\right)}} e^{\frac{1}{2} \partial_{s}} .
\end{aligned}
$$

Since the $\alpha$-operators are commuting, this case is not so interesting in applications (e.g., for $q$-models of the harmonic oscillators). A special case of the Askey-Wilson polynomials are the continuous $q$-Jacobi polynomials corresponding to the roots $a=q^{\alpha^{\prime} / 2+1 / 4}, b=q^{\alpha^{\prime} / 2+3 / 4}$, $c=-q^{\beta^{\prime} / 2+1 / 4}, d=-q^{\beta^{\prime} / 2+3 / 4}[7]$, then we can solve the problem 1 only in the case when $\alpha^{\prime}=\beta^{\prime}=-1 / 2$.

The next case is when one of the roots $z_{i}$ vanishes. This is the 0-Askey-Wilson polynomials or the continuous dual $q$-Hahn [7]. In this case the first condition (18) (equivalently (32)) holds only when

$$
\left(z_{1}, z_{2}, z_{3}\right)=\left(t, \frac{1}{2}-t, \frac{1}{4}\right), \quad t \in \mathbb{R} .
$$

With the above choice of $z_{i}$ it is impossible to fulfill the second condition (19) hence it is impossible to obtain a simple closed dynamical algebra.

\subsubsection{Continuous $q$-Laguerre polynomials}

Let now consider the case when the Askey-Wilson polynomials have two parameters equal to zero. In order that the condition (18) take place, the other two non-vanishing parameters should satisfy that their product is equal $q^{\frac{1}{2}}$. Under this condition $\varsigma=q^{-\frac{1}{2}}$. Then the second condition yields $\Lambda=\frac{4 C_{\sigma}(\sqrt{q}-1)}{k_{q}^{2}}$. Then, the Askey-Wilson Hamiltonian with two zero roots of $\sigma$ admits a factorization with a non-trivial dynamical algebra. An example of this family is the continuous 
$q$-Laguerre polynomials $P_{n}^{(a)}(x \mid q)[7], x(s)=\cos \theta$, when $a=-1 / 2$ for which the Hamiltonian has the form $(A(s)=1)$

$$
\begin{aligned}
\mathfrak{H}(s ; q)=-\frac{C_{\sigma}}{k_{q} \sin \theta}\left(\frac{\sqrt{\left(q^{s+1}-1\right)\left(q^{s+1}-q^{\frac{1}{2}}\right)\left(q^{-s}-1\right)\left(q^{-s}-q^{\frac{1}{2}}\right)}}{\sin \left(\theta+\frac{i}{2} \log q\right)} e^{-\partial_{s}}+\right. \\
\left.+\frac{\sqrt{\left(q^{s}-1\right)\left(q^{s}-q^{\frac{1}{2}}\right)\left(q^{1-s}-1\right)\left(q^{1-s}-q^{\frac{1}{2}}\right)}}{\sin \left(\theta-\frac{i}{2} \log q\right)} e^{\partial_{s}}\right)+ \\
-\frac{C_{\sigma}}{k_{q} \sin \theta}\left(\frac{\left(q^{s}-1\right)\left(q^{s}-q^{\frac{1}{2}}\right)}{\sin \left(\theta+\frac{i}{2} \log q\right)}+\frac{\left(q^{-s}-1\right)\left(q^{-s}-q^{\frac{1}{2}}\right)}{\sin \left(\theta-\frac{i}{2} \log q\right)}\right) I,
\end{aligned}
$$

and

$$
\begin{aligned}
& a_{1 / 2}^{\downarrow}(s ; q)=\frac{\sqrt{-C_{\sigma}}}{k_{q} \sin \theta}\left(e^{\frac{1}{2} \partial_{s}} \sqrt{\left(q^{s}-1\right)\left(q^{s}-q^{\frac{1}{2}}\right)}-e^{-\frac{1}{2} \partial_{s}} \sqrt{\left(q^{-s}-1\right)\left(q^{-s}-q^{\frac{1}{2}}\right)}\right), \\
& a_{1 / 2}^{\uparrow}(s ; q)=\frac{\sqrt{-C_{\sigma}}}{k_{q} \sin \theta}\left(\sqrt{\left(q^{s}-1\right)\left(q^{s}-q^{\frac{1}{2}}\right)} e^{-\frac{1}{2} \partial_{s}}-\sqrt{\left(q^{-s}-1\right)\left(q^{-s}-q^{\frac{1}{2}}\right)} e^{\frac{1}{2}} \partial_{s}\right) .
\end{aligned}
$$

Then for the functions

$$
\Phi_{n}(x(s))=\sqrt{\frac{\left(q^{\frac{1}{2}} ; q\right)_{n}\left(q, q^{\frac{1}{2}} ; q\right)_{\infty} \omega(s)}{(q ; q)_{n}}} 3_{\varphi_{2}}\left(\begin{array}{c}
q^{-n}, q^{-s}, q^{s} \\
q^{\frac{1}{2}}, 0
\end{array} \mid q ; q\right), \quad q^{s}=e^{i \theta}, \quad x(s)=\cos \theta
$$

we have the orthogonality $\int_{-1}^{1} \Phi_{n}(x ; q) \Phi_{m}(x ; q) d x=\delta_{n, m}$, and

$$
\mathfrak{H}(s ; q) \Phi_{n}(s ; q)=q\left(q^{-n}-1\right) \Phi_{n}(s ; q), \quad \mathfrak{H}(s ; q)=a_{1 / 2}^{\uparrow}(s ; q) a_{1 / 2}^{\downarrow}(s),
$$

and

$$
\left[a_{1 / 2}^{\downarrow}(s ; q), a_{1 / 2}^{\uparrow}(s)\right]_{q^{-1 / 2}}=\frac{4 C_{\sigma}(\sqrt{q}-1)}{k_{q}^{2}},
$$

Thus choosing $C_{\sigma}=-\frac{k_{q}^{2}}{4(1-\sqrt{q})}$ we obtain the relation $\left[a_{1 / 2}^{\downarrow}(s ; q), a_{1 / 2}^{\uparrow}(s)\right]_{q^{-1 / 2}}=I$.

The case of Askey-Wilson polynomials with three zero parameters is analogue to the case of one zero parameter and there is not possible to solve the problem 1. An example of this case are the continuous big $q$-Hermite polynomials [7].

\subsubsection{Continuous $q$-Hermite polynomials}

Finally, if one takes the Askey-Wilson polynomials with vanishing parameters $a, b, c, d$, this gives the continuous $q$-Hermite polynomials [7]. In this case $\sigma(z)=C_{\sigma} q^{2 z}$.

Let choose $A(s)=\sqrt{\nabla x_{1}(s)}$. Taking into account that this family is a special case of the Askey-Wilson polynomials when all parameters $a=b=c=d=0$, one directly obtains, by using $\varsigma=1 / q$, that in this case the Hamiltonian is given by

$$
\begin{gathered}
\mathfrak{H}(s ; q)=\frac{-1}{k_{q}^{2} \sqrt{\sin \theta}}\left(\frac{C_{\sigma} q}{\sin \left(\theta+\frac{i}{2} \log q\right) \sqrt{\sin (\theta+i \log q)}} e^{-\partial_{s}}+\frac{C_{\sigma} q}{\sin \left(\theta-\frac{i}{2} \log q\right) \sqrt{\sin (\theta-i \log q)}} e^{\partial_{s}}\right) \\
+\frac{1}{k_{q}^{2} \sin \theta}\left(\frac{C_{\sigma} q^{2 s}}{\sin \left(\theta+\frac{i}{2} \log q\right)}+\frac{C_{\sigma} q^{-2 s}}{\sin \left(\theta-\frac{i}{2} \log q\right)}\right) I,
\end{gathered}
$$


and the $\alpha$-operators

$$
\begin{aligned}
& a_{1 / 2}^{\downarrow}(s ; q)=e^{\frac{1}{2} \partial_{s}} \sqrt{\frac{C_{\sigma} q^{2 s}}{-k_{q}^{2} \sin \theta \sin \left(\theta+\frac{i}{2} \log q\right)}}-e^{-\frac{1}{2} \partial_{s}} \sqrt{\frac{C_{\sigma} q^{-2 s}}{-k_{q}^{2} \sin \theta \sin \left(\theta-\frac{i}{2} \log q\right)}} \\
& a_{1 / 2}^{\uparrow}(s ; q)=\sqrt{\frac{C_{\sigma} q^{2 s}}{-k_{q}^{2} \sin \theta \sin \left(\theta+\frac{i}{2} \log q\right)}} e^{-\frac{1}{2} \partial_{s}}-\sqrt{\frac{C_{\sigma} q^{-2 s}}{-k_{q}^{2} \sin \theta \sin \left(\theta-\frac{i}{2} \log q\right)}} e^{\frac{1}{2} \partial_{s}}
\end{aligned}
$$

are such that

$$
a^{\uparrow}(s ; q) a^{\downarrow}(s ; q)=\mathfrak{H}(s ; q) \quad \text { and } \quad\left[a^{\downarrow}(s ; q), a^{\uparrow}(s ; q)\right]_{1 / q}=\frac{4 C_{\sigma}}{k_{q}} .
$$

Notice that for getting the normalized commutation relations it is sufficient to choose $C_{\sigma}=k_{q} / 4$.

Another possible choice is $A(s)=1$ [12], hence a straightforward calculus shows that the two conditions in Theorem 3.4 are true if $\varsigma=q^{-1}$, thus $\Lambda=4 C_{\sigma} k_{q}^{-1}$. With this choice the orthogonality of the functions $\Phi_{n}$ is $\int_{-1}^{1} \Phi_{n}(s ; q) \Phi_{m}(s ; q) d x=\delta_{n, m}$. In this case, the Hamiltonian is equal to

$$
\mathfrak{H}(s ; q)=\frac{C_{\sigma} q}{k_{q}^{2}}\left\{\frac{e^{-\partial_{s}}}{\sin \theta \sin \left(\theta+\frac{i}{2} \ln q\right)}+\frac{e^{\partial_{s}}}{\sin \left(\theta-\frac{i}{2} \ln q\right) \sin \theta}-\frac{4}{\sqrt{q}}\left(1-\frac{1+q}{q+q^{-1}-2 \cos 2 \theta}\right) I\right\},
$$

and

$$
\begin{aligned}
& \mathfrak{a}^{\downarrow}(s ; q):=\mathfrak{a}_{1 / 2}^{\downarrow}(s)=\frac{\sqrt{-C_{\sigma}}}{k_{q} \sin \theta}\left(e^{\frac{1}{2} \partial_{s}} q^{s}-e^{-\frac{1}{2} \partial_{s}} q^{-s}\right), \\
& \mathfrak{a}^{\uparrow}(s ; q):=\mathfrak{a}_{1 / 2}^{\uparrow}(s)=\frac{\sqrt{-C_{\sigma}}}{k_{q} \sin \theta}\left(q^{s} e^{-\frac{1}{2} \partial_{s}}-q^{-s} e^{\frac{1}{2} \partial_{s}}\right) .
\end{aligned}
$$

In terms of these operators

$$
\mathfrak{H}(s ; q)=a^{\uparrow}(s ; q) a^{\downarrow}(s ; q) \quad \text { and } \quad\left[a^{\downarrow}(s ; q), a^{\uparrow}(s ; q)\right]_{q^{-1}}=\frac{4 C_{\sigma}}{k_{q}} .
$$

As before we now can choose $C_{\sigma}=k_{q} / 4$. This case was first considered in [13], see also [25].

\section{Appendix}

Here we present a simple proof of the Theorem 3.5 when $\alpha=0$. Notice that in this case $b(s ; q)=a^{\dagger}(s ; q)$, i.e., the operator $b$ is the adjoint of $a$.

Theorem 3.5a: Let $\left\{\Phi_{n}(s ; q)\right\}$ be the eigenfunctions of the operator $\mathfrak{H}(s ; q)$, corresponding to the eigenvalues $\left\{\lambda_{n}(q)\right\}$. Suppose that the $\mathfrak{H}(s ; q)$ admits the factorization (13). If the operators $a(s ; q)$ and $b(s ; q)$ in (13) satisfy the $q$-commutation relation $[a(s ; q), b(s ; q)]_{q}=I$, then the eigenvalues $\lambda_{n}(q)$ are $q$-linear or $q^{-1}$-linear functions of $n$, i.e., either $\lambda_{n}(q)=C_{1} q^{n}+C_{3}$ or $\lambda_{n}(q)=C_{2} q^{-n}+C_{3}$, respectively.

Proof: Obviously, the operator $\mathfrak{H}(s ; q)$ is diagonal in the basis consisting of its eigenfunctions $\Phi_{n}(s ; q)$. By hypothesis, this operator admits at the same time the factorization (13) in terms of $a(s ; q)$ and $b(s ; q)$. But according to proposition 3.2 the function $a(s ; q) \Phi_{n}(s ; q)$ is also the eigenfunction of $\mathfrak{H}(s ; q)$, associated with the eigenvalue $q^{-1}\left[\lambda_{n}(q)-1\right]$. Hence the $a(s ; q)$ is either the lowering operator or the raising operator. In the former case this means that

$$
q^{-1}\left[\lambda_{n}(q)-1\right]=\lambda_{n-1}(q)+C,
$$


from which it follows that $C_{2}=0$ (i.e., the spectrum $\left\{\lambda_{n}(q)\right\}$ is a $q$-linear one) and $C=$ $q^{-1}\left[(1-q) C_{3}-1\right]$. In latter case the corresponding relation is

$$
q^{-1}\left[\lambda_{n}(q)-1\right]=\lambda_{n+1}(q)+C
$$

which holds when $C_{1}=0$ (i.e., the spectrum is $q^{-1}$-linear) and $C=q^{-1}\left[(1-q) C_{3}-1\right]$. The proof of the theorem is thus complete.

Acknowledgments: The research of RAN has been partially supported by the Ministerio de Ciencias y Tecnología of Spain under the grant BFM 2003-06335-C03-01, the Junta de Andalucía under grant FQM-262. The participation of NMA in this work has been supported in part by the UNAM-DGAPA project IN102603-3 "Óptica Matemática" and the Junta de Andalucía under grant 2003/2. Finally, we are grateful to both the referees for their remarks and suggestions, which helped us to improve the exposition of our results.

\section{References}

[1] R. Álvarez-Nodarse and R.S. Costas-Santos, Factorization method for difference equations of hypergeometric-type on nonuniform lattices. J. Phys. A: Math. Gen 34 (2001), 5551-5569.

[2] A.F. Nikiforov and V.B. Uvarov, The Special Functions of Mathematical Physics, "Nauka", Moscow, 1978; Birkhäuser, Basel, 1988.

[3] A. F. Nikiforov, S. K. Suslov, and V. B. Uvarov, Classical Orthogonal Polynomials of a Discrete Variable. Springer Series in Computational Physics. Springer-Verlag, Berlin, 1991.

[4] A. F. Nikiforov and V. B. Uvarov, Polynomial Solutions of hypergeometric type difference equations and their classification. Integral Transforms and Special Functions. 1 (1993), 223-249.

[5] N. M. Atakishiyev, M. Rahman, and S. K. Suslov, On Classical Orthogonal Polynomials. Constructive Approximation 11 (1995), 181-226.

[6] G. Gasper and M. Rahman, Basic Hypergeometric Series. Cambridge University Press, Cambridge, 1990.

[7] R. Koekoek and R. F. Swarttouw, The Askey-scheme of hypergeometric orthogonal polynomials and its q-analogue. Reports of the Faculty of Technical Mathematics and Informatics, No. 98-17, Delft University of Technology, Delft, 1998.

[8] R. Álvarez-Nodarse and J. C. Medem, $q$-Classical polynomials and the $q$-Askey and NikiforovUvarov Tableaus. J. Comput. Appl. Math. 135 (2001) 197-223.

[9] N. M. Atakishiyev, Construction of the dynamical symmetry group of the relativistic harmonic oscillator by the Infeld-Hull factorization method. In Group Theoretical Methods in Physics, M. Serdaroglu and E. Inönü (Eds.), Lecture Notes in Physics, Springer-Verlag, 180, 1983, 393-396; Theor. Math. Phys. 56 (1984), 563-572.

[10] A. J. Macfarlane, On $q$-analogues of the quantum harmonic oscillator and the quantum group $S U_{q}(2)$. J. Phys. A: Math. Gen. 22 (1989), 4581-4588.

[11] L. C. Biedenharn, The quantum group $S U_{q}(2)$ and a $q$-analogue of the boson operators. J. Phys. A: Math. Gen. 22 (1989), L873-L878.

[12] N. M. Atakishiyev and S. K. Suslov, Difference analogs of the harmonic oscillator. Theoret. and Math. Phys. 85 (1991), 1055-1062.

[13] N. M. Atakishiyev and S. K. Suslov, A realization of the $q$-harmonic oscillator. Theoret. and Math. Phys. 87 (1991), 442-444. 
[14] R. Askey and S. K. Suslov, The $q$-harmonic oscillator and the Al-Salam and Carlitz polynomials. Lett. Math. Phys. 29 (1993), 123-132.

[15] R. Askey and S. K. Suslov, The $q$-harmonic oscillator and an analogue of the Charlier polynomials. J. Phys. A: Math. Gen. 26 (1993), L693-L698.

[16] N. M. Atakishiyev, A. Frank, and K. B. Wolf, A simple difference realization of the Heisenberg q-algebra. J. Math. Phys. 35 (1994), 3253-3260.

[17] N.M.Atakishiyev, E.I.Jafarov, Sh.M.Nagiyev, and K.B.Wolf, Meixner oscillators, Revista Mexicana de Física 44 No.3 (1998), 235-244.

[18] G.Bangerezako, Discrete Darboux transformation for discrete polynomials of hypergeometric type. J. Phys. A: Math. Gen. 31 (1998), 2191-2196.

[19] P. P. Kulish and E. V. Damaskinsky, On the $q$ oscillator and the quantum algebra $s u_{q}(1,1)$. J. Phys. A: Math. Gen. 23 (1990), L415-L419.

[20] N. Ja. Vilenkin and A.U. Klimyk, Representations of Lie Groups and Special Functions. Vol. I,II,III. Kluwer Academic Publishers. Dordrecht, 1992.

[21] A. S. Zhedanov, "Hidden symmetry" of the Askey-Wilson polynomials. Theor. Math. Phys. 89 (1992), 1146-1157.

[22] N. I. Akhiezer, The Classical Moment Problem and Some Related Questions in Analysis. Oliver and Boyd, Edinburg, 1965.

[23] J. A. Shohat and J. D. Tamarkin, The Problem of Moments. American Mathematical Society, Providence, Rhode Island, 1970.

[24] R. Álvarez-Nodarse and J. Arvesú, On the $q$-polynomials on the exponential lattice $x(s)=c_{1} q^{s}+c_{3}$. Integral Transforms and Special Functions. 8 (1999), 299-324.

[25] R. Álvarez-Nodarse, M.K. Atakishiyeva, and N. M. Atakishiyev. On a q-extension of the Hermite polynomials $H_{n}(x)$ with the continuous orthogonality property on $\mathbb{R}$, Bol. Soc. Mat. Mexicana 8 (2002), 127-139. 